Pacific Journal of Mathematics

DERIVATION ALGEBRAS OF FINITELY GENERATED WITT 


\title{
DERIVATION ALGEBRAS OF FINITELY GENERATED WITT RINGS
}

\author{
ROBERT W. FitzGERALD
}

\begin{abstract}
We consider derivations of an abstract Witt ring $R$. Denote the collection of derivations by $\operatorname{Der}(R)$; it is a Lie algebra under the usual bracket operation. The structure of $\operatorname{Der}(R)$ is closely related to the structure of the torsion part of $R$, which is the part least understood. After a lengthy computation of $\operatorname{Der}(R)$ for finitely generated Witt rings of elementary type, we classify the Witt rings in the following cases: (i) $\operatorname{Der}(R)=0$, (ii) $\operatorname{Der}(R)$ is a simple algebra, and (iii) the fundamental ideal of $R$ is not differentiable.
\end{abstract}

All Witt rings considered here will be finitely generated abstract Witt rings (in the sense of Marshall [5]). The most important examples are the Witt rings $W F$ of non-degenerate quadratic forms over a field $F$ with char $F \neq 2$ and $\dot{F} / \dot{F}^{2}$ a finite group. The basic problem is to classify these Witt rings. To date this has been done only for Witt rings that are small in some sense (e.g., the number of generators is $\leq 32$ ) and for torsion-free Witt rings. Indeed the part of a Witt ring $R$ that is least understood is its torsion ideal $R_{t}$.

We study here the derivations of a Witt ring $R$, namely, additive maps $D: R \rightarrow R$ such that $D(r s)=s D(r)+r D(s)$ for all $r, s \in R$. We denote by $\operatorname{Der}(R)$ the collection of all derivations of $R$. $\operatorname{Der}(R)$ is a Lie algebra, called the derivation algebra of $R$, under the usual bracket operation: if $D, D^{\prime} \in \operatorname{Der}(R)$ then $\left[D, D^{\prime}\right]=D \circ D^{\prime}-D^{\prime} \circ D \in \operatorname{Der}(R)$.

The usefulness of derivations appears to stem from the (easily checked) fact that the image of any derivation of $R$ lies in $R_{t}$. Thus the structure of the derivation algebra $\operatorname{Der}(R)$ sheds some light on the structure of $R_{t}$. We have obtained only some partial results however. We do classify the Witt rings in the following cases: (i) $\operatorname{Der}(R)=0$, (ii) $\operatorname{Der}(R)$ is a simple algebra, (iii) the fundamental ideal $I_{R}$ is not differentiable (i.e., $D\left(I_{R}\right) \not \subset I_{R}$ for some $D \in \operatorname{Der}(R)$ ), and (iv) every derivation on $R$ is integrable (but here we require some restrictions on $R$ ).

All of our classification results are special cases of a general classification of finitely generated Witt rings proposed by Marshall. We describe this. Start with the fundamental Witt rings $\mathbf{Z} / 2 \mathbf{Z}, \mathbf{Z} / 4 \mathbf{Z}, \mathbf{Z}$ and certain 
Witt rings of local type, namely, $L_{2 n, 0}, L_{2 n, 1}$ and $L_{2 n-1},(n \geq 2)$. The last three families arise as the Witt rings of suitable local fields (see [5] for details). We can form new Witt rings from old in two ways. If $R$ is a Witt ring and $\Delta$ is a (finite) group of exponent 2 then the group ring $R[\Delta]$ is again a Witt ring. If $R_{1}, R_{2}$ are Witt rings then the Witt product (or fibre product over $\mathbf{Z} / 2 \mathbf{Z})$ is $R_{1} \times_{w} R_{2}=\left\{\left(r_{1}, r_{2}\right) \mid r_{1} \in R_{1}, r_{2} \in R_{2}\right.$ and $\operatorname{dim} r_{1}$ $\left.\equiv \operatorname{dim} r_{2}(\bmod 2)\right\}$, which is also a Witt ring. A Witt ring is of elementary type if it can be built up from the fundamental Witt rings listed above by a sequence of group ring extensions and Witt products. The proposed classification is simply that every finitely generated Witt ring is of elementary type.

The first section of this paper presents elementary results and reduction theorems. The second section computes the derivation algebra for any Witt ring of elementary type. An important step here is deriving some short exact sequences relating $\operatorname{Der}\left(L_{2 n, 0}\right), \operatorname{Der}\left(L_{2 n, 1}\right)$ and $\operatorname{Der}\left(L_{2 n-1}\right)$.

The third section examines some examples. We give an example of two non-isomorphic Witt rings on 8 generators with Lie isomorphic derivation algebras. We also give an example of derivations arising naturally in the theory of quadratic forms. Let $F \subset K$ be a quadratic field extension and let $s_{*}$ denote the usual Scharlau transfer and $i_{*}$ denote the map on Witt rings induced by inclusion. Then, in many cases $i_{*} s_{*}$ : $W K \rightarrow W K$ is a derivation.

The fourth section is devoted to proving $L=\operatorname{Der}(R)$ is a simple algebra iff $R$ is a group ring over $\mathbf{Z} / 2 \mathbf{Z}$ and $L=W_{n}$, the generalized Witt algebra, for some $n$.

The fifth section examines when a derivation on a Witt ring $R$ is integrable (in the sense of Matsumura [6]). We show that if $R$ is of elementary type then every derivation is integrable iff $R$ is not a group ring extension of a ring of characteristic two.

The notation for Witt rings generally follows [5]. $R$ will always denote a finitely generated Witt ring. There is an associated group $G$ (of one dimensional forms) with distinguished element -1 . Every element of $R$ may be expressed as a form $\left\langle g_{1}, \ldots, g_{n}\right\rangle$ with each $g_{i} \in G$. There is also an associated linked quaternionic mapping $q: G \times G \rightarrow B$, where $B$ is some set. If there is a group $H$ and a linked quaternionic mapping $q$ : $H \times H \rightarrow B^{\prime}$ onto some set $B^{\prime}$, then there is induced a Witt ring which we will denote $W H$. $I_{R}$ denotes the fundamental ideal of $R$, that is, the collection of even dimensional forms.

If $r=\left\langle g_{1}, \ldots, g_{n}\right\rangle \in R$ then $D\left\langle g_{1}, \ldots, g_{n}\right\rangle$ (or $D_{R}\left\langle g_{1}, \ldots, g_{n}\right\rangle$ if the ring $R$ needs to be specified) is the set of elements of $G$ represented by $r$. 
This should be distinguished from $D\left(\left\langle g_{1}, \ldots, g_{n}\right\rangle\right)$-note the parentheses - which will indicate the image of $r$ under the derivation $D$.

We will write $\mathbf{Z}_{n}$ for $\mathbf{Z} / n \mathbf{Z}$. $\Delta_{n}$ will always denote a group of exponent 2 and order $2^{n}$. A universal round form in $R$ is a form $r \in R$ such that $g r=r$ for all $g$ in the associated group $G$. We let $\operatorname{ur}(R)$ denote the collection of all universal round forms; $\operatorname{ur}(R)$ is an ideal. If $I \subset R$ is an ideal, $\operatorname{ann}_{R} I$ denotes the annihilator of $I$ in $R$.

1. Reductions. We begin an elementary observation:

LeMma 1.1. Let $D \in \operatorname{Der}(R)$. Then:

(1) $D(1)=D(-1)=0$;

(2) $D(R) \subset \operatorname{ann}(\langle 1,1\rangle)$.

Proof. (1) $D(1)=D(1 \cdot 1)=D(1)+D(1)$, so $D(1)=0$. And $0=$ $D(0)=D(\langle 1,-1\rangle)=D(1)+D(-1)$, so $D(-1)=0$.

(2) It suffices to show $D(x) \in \operatorname{ann}(\langle 1,1\rangle)$ for all $x \in G$. Now $0=$ $D(1)=D(x \cdot x)=x D(x)+x D(x)$. Hence $\langle 1,1\rangle D(x)=0$.

It will frequently be easier to define a map on $G$ and show it extends to a derivation on $R$. The appropriate restrictions on the map on $G$ are in the following:

Definition. Let $R$ be a Witt ring and $G$ its associated group. A $G$-derivation is a map $d: G \rightarrow R$ such that

(i) $d(-1)=0$;

(ii) $d(x y)=x d(y)+y d(x)$, for all $x, y \in G$;

(iii) $d(x y)=d(x)+d(y)$ if $x \in D\langle 1, y\rangle$.

Let $\operatorname{Der}(G)$ denote the collection of $G$-derivations. Note that for $d \in \operatorname{Der}(G), d(1)=0$ and $d(G) \subset \operatorname{ann}(\langle 1,1\rangle)$ (namely, the proof of $(1.1)$ carries over).

Proposition 1.2. Every G-derivation induces a derivation on $R$. In particular, there is a bijection $\operatorname{Der}(G) \leftrightarrow \operatorname{Der}(R)$.

Proof. Let $d \in \operatorname{Der}(G)$ and define $D: R \rightarrow R$ by $D\left(\left\langle a_{1}, \ldots, a_{n}\right\rangle\right)=$ $d\left(a_{1}\right)+\cdots+d\left(a_{n}\right)$. Note that $D(\langle 1,-1\rangle)=0$. To show $D$ is welldefined it suffices to check on binary forms (cf. [5, p. 31]). Suppose $\left\langle x_{1}, x_{2}\right\rangle=\left\langle y_{1}, y_{2}\right\rangle$ in $R$. Then $x_{1} x_{2}=y_{1} y_{2}$ and $x_{1} y_{1} \in D\left\langle 1, x_{1} x_{2}\right\rangle$. Then 
$d\left(x_{2} y_{1}\right)=d\left(x_{1} y_{1} \cdot x_{1} x_{2}\right)=d\left(x_{1} y_{1}\right)+d\left(x_{1} x_{2}\right)$, and $d\left(x_{2} y_{1}\right)=d\left(x_{1} y_{2}\right)$. Expanding these equations yields:

$$
\begin{aligned}
& x_{1} d\left(y_{2}\right)+y_{2} d\left(x_{1}\right)=x_{1} d\left(y_{1}\right)+y_{1} d\left(x_{1}\right)+x_{1} d\left(x_{2}\right)+x_{2} d\left(x_{1}\right), \\
& x_{1}\left(d\left(y_{1}\right)+d\left(y_{2}\right)\right)=\left\langle y_{1}, y_{2},-x_{2}\right\rangle d\left(x_{1}\right)+x_{1} d\left(x_{2}\right), \\
& x_{1} D\left(\left\langle y_{1}, y_{2}\right\rangle\right)=x_{1} D\left(\left\langle x_{1}, x_{2}\right\rangle\right), \\
& D\left(\left\langle y_{1}, y_{2}\right\rangle\right)=D\left(\left\langle x_{1}, x_{2}\right\rangle\right) .
\end{aligned}
$$

(In the second step we have used $d(z)=-d(z)$ for any $z \in G$.)

Now $D$ is clearly additive. And

$$
\begin{array}{r}
D\left(\left\langle x_{1}, \ldots, x_{n}\right\rangle \cdot\left\langle y_{1}, \ldots, y_{m}\right\rangle\right)=\sum d\left(x_{i} y_{j}\right)=\sum\left(x_{i} d\left(y_{j}\right)+y_{j} d\left(x_{i}\right)\right) \\
=\left\langle x_{1}, \ldots, x_{n}\right\rangle D\left(\left\langle y_{1}, \ldots, y_{m}\right\rangle\right)+\left\langle y_{1}, \ldots, y_{m}\right\rangle D\left(\left\langle x_{1}, \ldots, x_{n}\right\rangle\right) .
\end{array}
$$

Hence $D \in \operatorname{Der}(R)$.

Lastly, suppose $D \in \operatorname{Der}(R)$ and set $d=\left.D\right|_{G}$. Then $d$ is a $G$-derivation. Namely, condition (i) holds by (1.1), condition (ii) holds by definition and if $x \in D\langle 1, y\rangle$ then $D(x)+D(x y)=D(x \cdot\langle 1, y\rangle)=D(\langle 1, y\rangle)$ $=D(y)$. Thus $d(x y)=d(x)+d(y)$, since $D(x)=-D(x)$ by (1.1).

REMARKS. (1) We will identity $G$-derivations with derivations on $R$.

(2) Combining conditions (ii) and (iii) for a $G$-derivation yields:

If $x \in D\langle 1, y\rangle$ then $\langle 1, x\rangle d(y)=\langle 1, y\rangle d(x)$. This will be used frequently.

Definition. Let $\beta$ be a Pfister form of $R$ such that $i_{G}(D(\beta)) \leq 2$ and $\langle 1,1\rangle \beta=0$. Let $H$ be a subgroup of index 2 in $G$ containing -1 and contained in $D(\beta)$. For $x, y \in G$ define:

$$
d(H, x \beta)(y)= \begin{cases}x \beta & \text { if } y \notin H, \\ 0 & \text { if } y \in H .\end{cases}
$$

The derivation (induced by) $d(H, x \beta)$ is the derivation of $H$ and $x \beta$.

We check this definition makes sense.

\section{LEMMA 1.3. $d(H, x \beta)$ is a derivation.}

Proof. Let $d$ denote $d(H, x \beta) ; d(-1)=0$. Let $y_{1}, y_{2} \in G$. We check conditions (ii) and (iii) for a $G$-derivation at the same time. Since we may switch the roles of $y_{1}, y_{2}$ there are three cases to consider:

Case 1. $y_{1}, y_{2} \in H$.

Here $d\left(y_{1}\right)=d\left(y_{2}\right)=d\left(y_{1} y_{2}\right)=0$. So $d\left(y_{1} y_{2}\right)=y_{1} d\left(y_{2}\right)+y_{2} d\left(y_{1}\right)$ $=d\left(y_{1}\right)+d\left(y_{2}\right)$. 
Case 2. $y_{1} \notin H, y_{2} \in H$.

Here $d\left(y_{1}\right)=x \beta, d\left(y_{2}\right)=0$ and $d\left(y_{1} y_{2}\right)=x \beta$. Note that since $y_{2} \in H \subset D(\beta), y_{2} x \beta=x \beta$. Thus we have:

$$
d\left(y_{1} y_{2}\right)=y_{1} d\left(y_{2}\right)+y_{2} d\left(y_{1}\right)=d\left(y_{1}\right)+d\left(y_{2}\right) \text {. }
$$

Case 3. $y_{1} \notin H, y_{2} \notin H$.

Here $y_{1} y_{2} \in H$ since $i_{G}(H)=2$. We hae $d\left(y_{1}\right)=x \beta=d\left(y_{2}\right)$ and $d\left(y_{1} y_{2}\right)=0=x \cdot\langle 1,1\rangle \beta=d\left(y_{1}\right)+d\left(y_{2}\right)$. Since $y_{1} y_{2} \in D(\beta), y_{1} d\left(y_{2}\right)$ $+y_{2} d\left(y_{1}\right)=y_{1}\left(d\left(y_{2}\right)+d\left(y_{1}\right)\right)=0=d\left(y_{1} y_{2}\right)$.

Proposition 1.4. $\operatorname{Der}(R)=0$ iff $R$ is reduced or $R \cong \mathbf{Z}_{2}$ or $R \cong \mathbf{Z}_{4}$. In particular, $R$ is of elementary type.

Proof. If $R$ is reduced then $\operatorname{Der}(R)=0$ by (1.1)(2), and if $R \cong \mathbf{Z}_{2}$ or $\mathbf{Z}_{4}$ then $G \subset\{ \pm 1\}$ and so $\operatorname{Der}(R)=0$ by (1.1)(1). Now suppose $R$ is not reduced, $\mathbf{Z}_{2}$ or $\mathbf{Z}_{4}$.

Since $R$ is not reduced we can choose $w \in D\langle 1,1\rangle \backslash\{1\}$. Then $\beta_{0}=\langle 1,-w\rangle \in \operatorname{ann}(\langle 1,1\rangle)$ and $\beta_{0} \neq 0$. We can find a non-zero Pfister form $\beta$, divisible by $\beta_{0}$, such that $D(\beta)=G$. Namely, suppose otherwise and choose a non-zero Pfister form $\beta$ with $D(\beta)$ maximal among those divisible by $\beta_{0}$ ( $\beta$ exists since $\left.|G|<\infty\right)$. If $D(\beta) \neq G$ take $x \in G \backslash D(\beta)$. Then $\langle 1,-x\rangle \beta \neq 0, \quad \beta_{0}$ divides $\langle 1,-x\rangle \beta$ and $-1,-x, D(\beta) \subset$ $D(\langle 1,-x\rangle \beta)$, so $\{1, x\} D(\beta) \subset D(\langle 1,-x\rangle \beta)$, contradicting the maximality of $\beta$.

Now $G \not \subset\{1,-1\}$, since $G \subset\{1,-1\}$ implies $R$ is isomorphic to $\mathbf{Z}_{2}$, $\mathbf{Z}_{4}$ or $\mathbf{Z}$ [5, p. 41-42] and $\mathbf{Z}$ is reduced. Choose $a \in G \backslash\{1,-1\}$ and choose $H$ a subgroup of index 2 in $G$ containing -1 but not $a$. Then $d(H, \beta)$ is a derivation (1.3) and non-zero since $d(H, \beta)(a)=\beta$. Thus $\operatorname{Der}(R) \neq 0$.

We begin the computation of $\operatorname{Der}(R)$ for $R$ of elementary type by decomposing $\operatorname{Der}(R)$ when $R$ is a group ring or a Witt product.

Proposition 1.5. Let $R=R_{0}\left[\Delta_{1}\right]$ with $\Delta=\{1, t\}$ and let $G_{0}$ be the group associated to $R_{0}$. Set:

$$
\begin{aligned}
& L_{0}=\left\{D \in \operatorname{Der}(R) \mid D(t)=0 \text { and } D\left(G_{0}\right) \subset R_{0}\right\}, \\
& L_{1}=\left\{D \in \operatorname{Der}(R) \mid D\left(G_{0}\right)=0 \text { and } D(t) \in R_{0}\right\} .
\end{aligned}
$$

Then: (1) $L_{0}$ is a subalgebra of $\operatorname{Der}(R)$ isomorphic to $\operatorname{Der}\left(R_{0}\right)$;

(2) $L_{1}$ is an abelian subalgebra isomorphic to $\operatorname{ann}_{R_{0}}(\langle 1,1\rangle)$;

(3) $\operatorname{Der}(R)=L_{0} \oplus t L_{0} \oplus L_{1} \oplus t L_{1}$. 
Proof. (1) $L_{0}$ is closed under addition and if $D_{1}, D_{2} \in L_{0}$ then $\left(D_{1} \circ D_{2}\right)(t)=0$ and $\left(D_{1} \circ D_{2}\right)\left(G_{0}\right) \subset D_{1}\left(R_{0}\right) \subset R_{0}$. So $L_{0}$ is a subalgebra. If $D \in L_{0}$ then $\left.D\right|_{R_{0}} \in \operatorname{Der}\left(R_{0}\right)$. Let $d \in \operatorname{Der}\left(R_{0}\right)$ and define $D\left(r_{0}+t r_{1}\right)=d\left(r_{0}\right)+t d\left(r_{1}\right)$, for $r_{0}, r_{1} \in R_{0}$. This is well-defined since each $r \in R$ uniquely determines $r_{0}, r_{1} \in R_{0}$ with $r=r_{0}+t r_{1}$. It is straightforward to check $D \in \operatorname{Der}(R)$. Since $D(t)=t d(1)=0$ and $d\left(G_{0}\right)$ $\subset R_{0}$ we have $D \in L_{0}$. Also $\left.D\right|_{R_{0}}=d$ and so $L_{0}$ is isomorphic to $\operatorname{Der}\left(R_{0}\right)$.

(2) $L_{1}$ is closed under addition and if $D_{1}, D_{2} \in L_{1}$ then $\left(D_{1} \circ D_{2}\right)(G)$ $=D_{1}\left(D_{2}\left(t G_{0}\right)\right) \subset D_{1}\left(R_{0}\right)=0$. Thus $L_{1}$ is an abelian subalgebra.

For $w \in \operatorname{ann}_{R_{0}}(\langle 1,1\rangle)$ define $d(w): G \rightarrow R$ by $d(w)\left(g_{0}\right)=0$ and $d(w)\left(g_{0} t\right)=g_{0} w$, for all $g_{0} \in G_{0}$. We check that $d(w)$ is a derivation. First, $d(w)(-1)=0$. Next, let $x, y \in G$. If $x, y \in G_{0}$ then $y d(w)(x)+$ $x d(w)(y)=0+0=d(w)(x y)$. If $x=g_{0} t \in t G_{0}, y \in G_{0}$ then $y d(w)(x)$ $+x d(w)(y)=y g_{0} w=d(w)(x y)$. And if $x=g_{0} t, y=g_{1} t$, with $g_{0}, g_{1} \in$ $G_{0}$ then $y d(w)(x)+x d(w)(y)=g_{0} g_{1} t w+g_{0} g_{1} t w=0=d(w)(x y)$, since $\langle 1,1\rangle w=0$.

Now suppose $x, y \in G$ and $x \in D\langle 1, y\rangle$. Then either $x, y \in G_{0}$ or $x=y \in t G_{0}$, since $t$ is 2-sided rigid [5, 5.19]. If $x, y \in G_{0}$ then $d(w)(x)$ $+d(w)(y)=0=d(w)(x y)$. And if $x=y$ then $d(w)(x)+d(w)(y)=$ $\langle 1,1\rangle d(w)(x)=0=d(w)(x y)$. Thus $d(w)$ is a derivation.

We have inverse homomorphisms $L_{1} \rightarrow \operatorname{ann}_{R_{0}}(\langle 1,1\rangle)$ by $D \mapsto D(t)$ and $\operatorname{ann}_{R_{0}}(\langle 1,1\rangle) \rightarrow L_{1}$ by $w \mapsto d(w)$. Hence $L_{1}$ is isomorphic to $\operatorname{ann}_{R_{0}}(\langle 1,1\rangle)$.

(3) It suffices to show $\operatorname{Der}(R)=L_{0}+t L_{0}+L_{1}+t L_{1}$ since the sum can easily be shown to be direct. Let $D \in \operatorname{Der}(R)$. If $r \in R_{0}$ there exist unique $r_{1}, r_{2} \in R_{0}$ such that $D(r)=r_{1}+t r_{2}$. Let $d_{1}(r)=r_{1}$ and $d_{2}(r)=$ $r_{2}$. Extend these maps to $R$ by setting $d_{i}\left(r+r^{\prime} t\right)=d_{i}(r)+t d_{i}\left(r^{\prime}\right)$, where $i=1,2$ and $r, r^{\prime} \in R_{0}$. Also, by (1.1), there exist unique $w_{1}, w_{2} \in$ ann $_{R_{0}}(\langle 1,1\rangle)$ such that $D(t)=w_{1}+t w_{2}$. Then $D=d_{1}+t d_{2}+d\left(w_{1}\right)+$ $t d\left(w_{2}\right)$, where the $d\left(w_{i}\right), i=1,2$, are the derivations defined in (2). So it suffices to show $d_{1}, d_{2} \in L_{0}$.

Now $d_{1}$ and $d_{2}$ are additive and $d_{i}(t)=0, d_{i}\left(G_{0}\right) \subset R_{0}(i=1,2)$. Let $r, r^{\prime} \in R_{0}$. Then:

$$
\begin{aligned}
D\left(r r^{\prime}\right) & =r D\left(r^{\prime}\right)+r^{\prime} D(r), \\
d_{1}\left(r r^{\prime}\right)+t d_{2}\left(r r^{\prime}\right) & =r d_{1}\left(r^{\prime}\right)+t r d_{2}\left(r^{\prime}\right)+r^{\prime} d_{1}(r)+t r^{\prime} d_{2}(r), \\
d_{i}\left(r r^{\prime}\right) & =r d_{i}\left(r^{\prime}\right)+r^{\prime} d_{i}(r), \quad(i=1,2) .
\end{aligned}
$$

Hence $\left.d_{i}\right|_{R_{0}} \in \operatorname{Der}\left(R_{0}\right)$ and, as in (1), $d_{i} \in L_{0}(i=1,2)$.

Corollary 1.6. $\left|\operatorname{Der}\left(R_{0}\left[\Delta_{1}\right]\right)\right|=\left|\operatorname{Der}\left(R_{0}\right)\right|^{2}\left|\operatorname{ann}_{R_{0}}(\langle 1,1\rangle)\right|^{2}$. 
Definition. An ideal $I \subset R$ is differentiable if $D(I) \subset I$ for all $D \in \operatorname{Der}(R)$.

THEOREM 1.7. $I_{R}$ is not differentiable iff $\operatorname{char}(R)=2$ and $R$ is a group ring.

Proof. ( $\rightarrow$ ). Let $r=\left\langle a_{1}, \ldots, a_{2 n}\right\rangle$ and $d \in \operatorname{Der}(R)$ such that $D(r) \notin$ $I_{R}$. Then for some $a_{i}$, say $a_{1}, D\left(a_{1}\right) \notin I_{R}$. By $(1.1),\langle 1,1\rangle D\left(a_{1}\right)=0$. Since $\operatorname{dim} D\left(a_{1}\right)$ is odd, $\langle 1,1\rangle=0$ [4, p. 250] and so $\operatorname{char}(R)=2$. Now let $x \in D\left\langle 1, a_{1}\right\rangle$. Then

$$
\left\langle 1, a_{1}\right\rangle D(x)=\langle 1, x\rangle D\left(a_{1}\right)
$$

Since $D\left(a_{1}\right)$ is odd dimensional, the discriminant of $\langle 1, x\rangle D\left(a_{1}\right)$ is $x$. But the discriminant of $\left\langle 1, a_{1}\right\rangle D(x)$ is 1 or $a_{1}$. Hence $x=1$ or $a_{1}$. So $D\left\langle 1, a_{1}\right\rangle=\left\{1, a_{1}\right\}$.

Further, $D\left(a_{1}\right)=D\left(-a_{1}\right) \notin I_{R}$ so the same argument shows $D\left\langle 1,-a_{1}\right\rangle=\left\{1,-a_{1}\right\}$. Hence $a_{1}$ is two-sided rigid and by [5, 51.9] $R$ is a group ring.

$(\leftarrow)$ Let $R=R_{0}\left[\Delta_{1}\right]$ where $\Delta_{1}=\{1, t\}$; note that $t$ is two-sided rigid. Now $\operatorname{char}(R)=2$ implies $1 \in \operatorname{ann}_{R_{0}}(\langle 1,1\rangle)$. Let $D=t d(1)$, where $d(1)$ is the derivation constructed in (1.5) with $d(1)\left(R_{0}\right)=0$ and $d(1)(t)$ $=\langle 1\rangle$. Then $D(\langle 1,1\rangle)=\langle t\rangle \notin I_{R}$.

We next consider Witt products. Let $R=R_{1} \times{ }_{w} R_{2}$ with corresponding groups $G=G_{1} \times G_{2}$. Let $L=\operatorname{Der}(R)$. We form the following subsets of $L$ :

$$
\begin{aligned}
& L_{1}=\left\{D \in L \mid D\left(1 \times G_{2}\right)=0, D\left(G_{1} \times 1\right) \subset I_{R_{1}} \times 0\right\}, \\
& L_{2}=\left\{D \in L \mid D\left(G_{1} \times 1\right)=0, D\left(1 \times G_{2}\right) \subset 0 \times I_{R_{2}}\right\}, \\
& E_{1}=\left\{D \in L \mid D\left(1 \times G_{2}\right)=0, D\left(G_{1} \times 1\right) \subset 0 \times I_{R_{2}}\right\}, \\
& E_{2}=\left\{D \in L \mid D\left(G_{1} \times 1\right)=0, D\left(1 \times G_{2}\right) \subset I_{R_{1}} \times 0\right\} .
\end{aligned}
$$

Suppose $\operatorname{char}(R) \neq 2 . R_{1}$ and $R_{2}$ cannot both have characteristic 2; we will assume $\operatorname{char}\left(R_{1}\right) \neq 2$. Then $(-1,1) \neq(1,1)$ in $G$. Fix a subgroup $B$ of index 2 in $G$ with $(-1,1) \notin B$. Set:

$$
F=\{D \in L \mid D(B)=0\} .
$$


Proposition 1.8. Let $R=R_{1} \times{ }_{w} R_{2}$ and $L=\operatorname{Der}(R)$. With the notations given above, we have:

(1) $L_{i}$ is a subalgebra isomorphic to

$$
\left\{D \in \operatorname{Der}\left(R_{i}\right) \mid D\left(R_{0}\right) \subset I_{R_{l}}\right\} \text { for } i=1,2 .
$$

(2) $E_{1}$ and $E_{2}$ are abelian subalgebras and

$$
\begin{aligned}
& E_{1} \cong \operatorname{Hom}\left(G_{1} /\{ \pm 1\}, \operatorname{ur}\left(R_{2}\right)\right) \\
& E_{2} \cong \operatorname{Hom}\left(G_{2} /\{ \pm 1\}, \operatorname{ur}\left(R_{1}\right)\right) .
\end{aligned}
$$

(3) If $\operatorname{char}(R) \neq 2$ then $F$ is a subalgebra isomorphic (as a group) to $\operatorname{ur}(R)$.

(4) If $\operatorname{char}(R) \neq 2$ then $L=L_{1} \oplus L_{2} \oplus E_{1} \oplus E_{2} \oplus F$.

(5) If $\operatorname{char}(R)=2$ then $L=L_{1} \oplus L_{2} \oplus E_{1} \oplus E_{2}$.

Proof. (1) Straightforward.

(2) We prove the result for $E_{1}$; the case for $E_{2}$ is similar. Let $D, D^{\prime} \in E_{1}$. Then $\left(D \circ D^{\prime}\right)(G) \subset D\left(0 \times I_{R_{2}}\right)=0$. Thus $E_{1}$ is an abelian subalgebra. Now $D(-1,1)=D(1,-1)=0$ and $D(G) \subset 0 \times \operatorname{ur}\left(R_{2}\right)$. Namely, if $h \in G_{1}$ and $k \in G_{2}$ then since $(1, k) \in D\langle(1,1),-(h, 1)\rangle$ we have

$$
\langle(1,1),-(1, k)\rangle D(h, 1)=\langle(1,1),-(h, 1)\rangle D(1, k)=0 .
$$

So if $D(h, 1)=(0, r)$, with $r \in I_{R_{2}}$, then $\langle 1,-k\rangle r=0$ for all $k \in G_{2}$ and so $r \in \operatorname{ur}\left(R_{2}\right)$.

We thus have $D(-1,1)=0$ and $D\left(G_{1} \times 1\right) \subset 0 \times \operatorname{ur}\left(R_{2}\right)$. So $D$ induces a map $e: G_{1} /\{ \pm 1\} \rightarrow \operatorname{ur}\left(R_{2}\right)$. Lastly,

$$
\begin{aligned}
0 \times e\left(h h^{\prime}\right) & =D\left(h h^{\prime}, 1\right)=(h, 1) D\left(h^{\prime}, 1\right)+\left(h^{\prime}, 1\right) D(h, 1) \\
& =D\left(h^{\prime}, 1\right)+D(h, 1)=0 \times e\left(h^{\prime}\right)+0 \times e(h) .
\end{aligned}
$$

Hence $e \in \operatorname{Hom}\left(G_{1} /\{ \pm 1\}, \operatorname{ur}\left(R_{2}\right)\right)$.

Conversely, let $e \in \operatorname{Hom}\left(g_{1} /\{ \pm 1\}, \operatorname{ur}\left(R_{2}\right)\right) ; e$ lifts to a unique map $\bar{e}$ in $\operatorname{Hom}\left(G_{1}, \operatorname{ur}\left(R_{2}\right)\right)$ sending -1 to 0 . Define $D\left(g_{1}, g_{2}\right)$ to be $\left(0, \bar{e}\left(g_{1}\right)\right)$, for all $g_{1} \in G_{1}, g_{2} \in G_{2}$. Then $D(-1)=0$ and

$$
\begin{aligned}
& D\left(g_{1} g_{1}^{\prime}, g_{2} g_{2}^{\prime}\right)=\left(0, \bar{e}\left(g_{1} g_{1}^{\prime}\right)\right)=\left(0, \bar{e}\left(g_{1}\right)\right)+\left(0, \bar{e}\left(g_{1}^{\prime}\right)\right) \\
& \quad=D\left(g_{1}, g_{2}\right)+D\left(g_{1}^{\prime}, g_{2}^{\prime}\right)=\left(g_{1}^{\prime}, g_{2}^{\prime}\right) D\left(g_{1}, g_{2}\right)+\left(g_{1}, g_{2}\right) D\left(g_{1}^{\prime}, g_{2}^{\prime}\right),
\end{aligned}
$$

since $D(G) \subset 0 \times \operatorname{ur}\left(R_{2}\right)$. By (1.2) $D \in L$ and clearly $D$ induces $e$.

(3) That $F$ is a subalgebra follows from the definitions. We map $\operatorname{ur}(R) \rightarrow F$ by $w \mapsto d(B, w)$, where $d(B, w)$ is the derivation of $B$ and $w$. The map is additive and injective. To show the map is surjective we first 
prove the

Claim. If $D \in L$ then $D(-1,1) \in \operatorname{ur}(R)$.

Let $h \in G_{1}$ and let $D(h, 1)=\left(r_{1}, r_{2}\right)$ with $r_{i} \in R_{i}$. Then $(h, 1) \in$ $D\langle(1,1), \quad(-1,1)\rangle \quad$ implies $\langle(1,1), \quad(h, 1)\rangle D(-1,1)=$ $\langle(1,1), \quad(-1,1)\rangle D(h, 1)=\left(\langle-1,1\rangle r_{1},\langle 1,1\rangle r_{2}\right)=0$, using (1.1). Similarly, if $k \in G_{2}$ then $(1, k) \in D\langle(1,1),(1,-1)\rangle, D(1,-1)=$ $D(-1,1)$ and $\langle(1,1),(1, k)\rangle D(-1,1)=\langle(1,1),(1,-1)\rangle D(1, k)=0$. Thus $\langle 1, g\rangle D(-1,1)=0$ for all $g \in G_{1} \times 1 \cup 1 \times G_{2}$ and hence for all $g \in G$. Then $D(-1,1) \in \operatorname{ur}(R)$ as claimed.

Now if $D \in F$ and $D(-1,1)=w$ then for any $b \in G, D(b)=0$ and $(b \cdot(-1,1))=b w=w$, since $w \in \operatorname{ur}(R)$. Hence $D=d(B, w)$ with $w \in$ $\operatorname{ur}(R)$ and the map is surjective.

(4), (5) It is easily verified that the sums involved are direct. Let $D \in L$. If $D(-1,1) \neq 0$ then $\operatorname{char}(R) \neq 2$ and $D(-1,1) \in \operatorname{ur}(R)$ by the claim in (3). By (3) we can find $D_{5} \in F$ such that $D(-1,1)=D_{5}(-1,1)$. It thus remains to show that if $D \in L$ and $D(-1,1)=0$ then $D \in L_{1}+$ $L_{2}+E_{1}+E_{2}$.

The only Witt product that is also a group ring is $\mathbf{Z} \times{ }_{w} \mathbf{Z}[\mathbf{5}, 5.22]$ which is reduced. Hence by $(1.7), D(G) \subset I_{R}$. Let $\pi_{1}, \pi_{2}$ be the projections from $I_{R}$ to $I_{R_{1}} \times 0,0 \times I_{R_{2}}$, respectively. Let $\rho_{1}, \rho_{2}$ be the projections from $G$ to $G_{1} \times 1,1 \times G_{2}$, respectively. Set $D_{1}=\pi_{1} D \rho_{1}, D_{2}=$ $\pi_{2} D \rho_{2}, D_{3}=\pi_{2} D \rho_{1}$ and $D_{4}=\pi_{1} D \rho_{2}$. Then we claim $D_{1} \in L_{1}, D_{2} \in L_{2}$, $D_{3} \in E_{1}$ and $D_{4} \in E_{2}$. We check this only for $D_{1}$ and $D_{3}$.

For $g, g^{\prime} \in G$,

$$
\begin{aligned}
D_{1}\left(g g^{\prime}\right) & =\pi_{1} D\left(\rho_{1}(g) \rho_{1}\left(g^{\prime}\right)\right) \\
& =\pi_{1}\left(\rho_{1}\left(g^{\prime}\right) D\left(\rho_{1}(g)\right)+\rho_{1}(g) D\left(\rho_{1}\left(g^{\prime}\right)\right)\right) \\
& =\rho_{1}\left(g^{\prime}\right) D_{1}(g)+\rho_{1}(g) D_{1}\left(g^{\prime}\right)=g^{\prime} D_{1}(g)+g D_{1}\left(g^{\prime}\right),
\end{aligned}
$$

since $D_{1}(G) \subset I_{R_{1}} \times 0$ implies $(1, y) D(x)=D(x)$ for any $y \in G_{2}, x \in G$. If $g \in D\left\langle 1, g^{\prime}\right\rangle$ then $\rho_{1}(g) \in D\left\langle 1, \rho_{1}\left(g^{\prime}\right)\right\rangle$ and so

$$
D_{1}\left(g g^{\prime}\right)=\pi_{1}\left(D\left(\rho_{1}(g)\right)+D\left(\rho_{2}\left(g^{\prime}\right)\right)\right)=D_{1}(g)+D_{1}\left(g^{\prime}\right) \text {. }
$$

Thus $D_{1} \in L_{1}$.

Now

$$
\begin{aligned}
D_{3}\left(g g^{\prime}\right) & =\pi_{2}\left(D\left(\rho_{1}(g)\right) \rho_{1}\left(g^{\prime}\right)\right) \\
& =\pi_{2}\left(\rho_{1}\left(g^{\prime}\right) D\left(\rho_{1}(g)\right)+\rho_{1}(g) D\left(\rho_{1}\left(g^{\prime}\right)\right)\right)=D_{3}(g)+D_{3}\left(g^{\prime}\right) .
\end{aligned}
$$


We thus only need to show that $g^{\prime} D_{3}(g)=D_{3}(g)$ for all $g, g^{\prime} \in G$. Fix $g \in G$. Since $D_{3}(g) \subset 0 \times I_{R_{2}}, g^{\prime} D_{3}(g)=D_{3}(g)$ if $g^{\prime} \in G_{1} \times 1$. If $g^{\prime}=$ $\left(1, k^{\prime}\right) \in 1 \times G_{2}$ then $g^{\prime} \in D\left\langle 1,-\rho_{1}(g)\right\rangle$ so $\left\langle 1,-g^{\prime}\right\rangle D\left(\rho_{1}(g)\right)=$ $\left\langle 1,-\rho_{1}(g)\right\rangle D\left(g^{\prime}\right)$. Now $\pi_{1}\left(\left\langle 1,-g^{\prime}\right\rangle D\left(\rho_{1}(g)\right)\right)=\langle 1,-1\rangle\left(\pi_{1} D \rho_{1}\right)(g) \times 0$ $=0$. And

$$
\begin{aligned}
\pi_{2}\left(\left\langle 1,-g^{\prime}\right\rangle D\left(\rho_{1}(g)\right)\right) & =\pi_{2}\left(\left\langle 1,-\rho_{1}(g)\right\rangle D\left(g^{\prime}\right)\right) \\
& =0 \times\langle 1,-1\rangle\left(\pi_{2} D\right)\left(g^{\prime}\right)=0 .
\end{aligned}
$$

Hence $g^{\prime} D\left(\rho_{1}(g)\right)=D\left(\rho_{1}(g)\right)$ if $g^{\prime} \in 1 \times G_{2}$. That is, we have $g^{\prime} D_{3}(g)$ $=D_{3}(g)$ for all $g^{\prime} \in G_{1} \times 1 \cup 1 \times G_{2}$ and hence for all $g^{\prime} \in G$. Thus, $D_{3} \in E_{1}$.

Lastly, we show $D=D_{1}+D_{2}+D_{3}+D_{4}$. For any $g \in g$. $\rho_{1}(g) \in$ $D\left\langle 1,-\rho_{2}(g)\right\rangle$. So $D(g)=D\left(\rho_{1}(g) \rho_{2}(g)\right)=D\left(\rho_{1}(g)\right)+D\left(\rho_{2}(g)\right)$. That is,

$$
\begin{aligned}
D & =D \rho_{1}+D \rho_{2}=\pi_{1} D \rho_{1}+\pi_{2} D \rho_{1}+\pi_{1} D \rho_{2}+\pi_{2} D \rho_{2} \\
& =D_{1}+D_{3}+D_{4}+D_{2} .
\end{aligned}
$$

The simplest case of (1.8) yields

Corollary 1.9. Suppose char $R_{1} \neq 2$, and $\operatorname{char} R_{2} \neq 2$. Let $R=$ $R_{1} \times_{w} R_{2}$ and let $G$ be the group associated to $R$. Then taking $\mathbf{Z}_{2}$-dimensions:

$\operatorname{dim} \operatorname{Der}(R)=\operatorname{dim} \operatorname{Der}\left(R_{1}\right)+\operatorname{dim} \operatorname{Der}\left(R_{2}\right)+(\operatorname{dim} G)(\operatorname{dim} u r(R))$.

Proof. We use the notations of (1.8). By (1.7), $L_{i}=\operatorname{der}\left(R_{i}\right)$ for $i=1,2$. We have by (1.8)

$$
\begin{aligned}
\operatorname{dim} E_{1} & =(\operatorname{dim} G-1) \operatorname{dim} u r\left(R_{1}\right) \\
\operatorname{dim} E_{2} & =(\operatorname{dim} G-1) \operatorname{dim} u r\left(R_{2}\right) \\
\operatorname{dim} F & =\operatorname{dim} u r\left(R_{1}\right)+\operatorname{dim} u r\left(R_{2}\right)
\end{aligned}
$$

(This last since $\operatorname{ur}(R)=\operatorname{ur}\left(R_{1}\right) \times \operatorname{ur}\left(R_{2}\right)$ ). The decomposition $\operatorname{Der}(R)=$ $L_{1} \oplus L_{2} \oplus E_{1} \oplus E_{2} \oplus F$ then yields the result.

2. Derivation algebras for fundamental Witt rings. To complete the computation of $\operatorname{Der}(R)$ for $R$ of elementary type we need to consider derivations on the fundamental Witt rings. These Witt rings are (cf. [5, 5.24]) $\mathbf{Z}, \mathbf{Z}_{2}, \mathbf{Z}_{4}$ and $L_{2 n, 0}, L_{2 n, 1}, L_{2 n-1}$ for $n \geq 2$. The latter three classes are Witt rings of local type. 
By (1.4), $\operatorname{Der}(R)=0$ for $R=\mathbf{Z}_{1} \mathbf{Z}_{2}$ and $\mathbf{Z}_{4}$. So for the rest of this section we will consider Witt rings of local type. Such Witt rings possess a unique non-trivial 2-fold Pfister form, to be denoted $\rho$ throughout this section. Further, for each $x \neq-1, D\langle 1, x\rangle$ is a subgroup of index 2 in $G$. The converse also holds, which we separate for future reference:

LEMma 2.1. Let $R$ be a Witt ring of local type with (finite) group $G$. For every subgroup $H$ of index 2 in $G$ there exists an $x \in G$ with $H=$ $D\langle 1, x\rangle$.

Proof. There are $|G|-1$ subgroups of index 2 in $G$. There are $|G|-1$ elements $x \in G \backslash\{-1\}$, each of which yields a (distinct) subgroup $D\langle 1, x\rangle$ of index 2 . Hence the result holds.

Lemma 2.2. Let $R$ be a Witt ring of local type with group $G$. Assume $R \neq \mathbf{Z}$.

(1) If $\operatorname{char}(R) \neq 2$ then $\operatorname{ann}_{R}\langle 1,1\rangle=\{0, \rho\} \cup\{\langle 1, x\rangle, y\langle 1, x\rangle \mid-x$ $\in D\langle 1,1\rangle \backslash\{0\}$ and $y \notin D\langle 1, x\rangle\}$. In particular, $\left|\operatorname{ann}_{R}\langle 1,1\rangle\right|=|G|$.

(2) If $\operatorname{char}(R)=2$ then $\operatorname{ann}_{R}\langle 1,1\rangle \cap I_{R}=\{0, \rho\} \cup\{\langle 1, x\rangle$, $y\langle 1, x\rangle \mid x \in G \backslash\{1\}, y \notin D\langle 1, x\rangle\}$. In particular, $\left|\operatorname{ann}_{R}\langle 1,1\rangle \cap I_{R}\right|=$ $2|G|$.

Proof. If $\operatorname{char}(R) \neq 2$ then $\operatorname{ann}_{R}\langle 1,1\rangle \subset I_{R}$. Thus it suffices to find $\operatorname{ann}_{R}\langle 1,1\rangle \cap I_{R}$ in both cases. We will show $I_{R}$ consists of $0, \rho$ and binary forms. From this (1) and (2) follow quickly.

Let $q \in I_{R}$, and let $d=d(q)$. If $d=1$ then $q \in I_{R}^{2}=\{0, \rho\}$. Otherwise, we may write $q=\langle 1,-d\rangle+q_{0}$, with $q_{0} \in I_{R}^{2}$. If $q_{0}=0$ we are done. If $q_{0}=\rho$, let $e \notin D\langle 1,-d\rangle$. Then $\langle\langle-d,-e\rangle\rangle=\rho$ and we obtain

$$
q=\langle 1,-d\rangle \cdot\langle 1,1,-e\rangle=e \cdot\langle 1,-d\rangle+\langle\langle 1,-e,-d\rangle\rangle=e \cdot\langle 1,-d\rangle,
$$
since $\langle\langle 1,-e,-d\rangle\rangle \in I_{r}^{3}=0$.

LEMma 2.3. Let $R$ be a Witt ring of local type with group $G$ and $\operatorname{char}(R) \neq 2$. Let $D \in \operatorname{Der}(R)$ and $g \in G$. If $D(g)=0$ then $\langle 1,-g\rangle D(x)=0$ for all $x \in G$.

Proof. Let $x \in G$. By (1.1), $D(x) \in \operatorname{ann}_{R}\langle 1,1\rangle$ and the result is clear if $D(x)=0$ or $\rho$. (Note that $D \equiv 0$ if $R=\mathbf{Z}$.) By (2.2) we may thus assume $D(x)=\langle 1,-y\rangle$ for some $y \in D\langle 1,1\rangle$. If $x \in D\langle 1,-g\rangle$ then 
$\langle 1,-g\rangle D(x)=\langle 1,-x\rangle D(g)=0$. We thus suppose $x \notin D\langle 1,-g\rangle$; in particular, $g \neq 1$.

Now $\operatorname{char}(R) \neq 2$ implies $D\langle 1,1\rangle \neq G$. We claim that $D\langle 1, x\rangle \cap$ $D\langle 1, g\rangle \backslash D\langle 1,1\rangle \neq \varnothing$. Otherwise, $D\langle 1, x\rangle \cap D\langle 1, g\rangle \subset D\langle 1,1\rangle$. But $D\langle 1, x\rangle \cap D\langle 1, g\rangle$ is a subgroup of index 4 in $G$, thus contained in only three subgroups of index 2 . These three subgroups are $D\langle 1, x\rangle, D\langle 1, g\rangle$ and $D\langle 1,-x g\rangle$. This implies that one of $x, g,-x g$ is 1 . Each possibility contradicts our previous assumptions, which proves the claim.

Let $z \in D\langle 1, x\rangle \cap D\langle 1, g\rangle \backslash D\langle 1,1\rangle$. Then $z \notin D\langle 1,-g\rangle$ lest $z \in$ $D\langle 1, g\rangle \cap D\langle 1,-g\rangle \subset D\langle 1,1\rangle$. Also, since $x \notin D\langle 1,-g\rangle$ and $D\langle 1,-g\rangle$ is a group of index 2 , we have $x z \in D\langle 1,-g\rangle$. Lastly, $x, z \in$ $D\langle 1, x\rangle$ implies $x z \in D\langle 1, x\rangle \cap D\langle 1,-g\rangle \subset D\langle 1, g x\rangle$, so $-g x \in$ $D\langle 1,-x z\rangle$. We have:

$$
\begin{aligned}
\langle 1,-x z\rangle D(x g) & =\langle 1,-x g\rangle D(x z), \\
\langle 1,-x z\rangle D(x) & =\langle 1,-x g\rangle D(x)+\langle 1,-x g\rangle D(z),
\end{aligned}
$$

since $D(x g)=g D(x),\langle 1,-x z\rangle D(x)=0$ or $\rho$ and so $g\langle 1,-x z\rangle D(x)=$ $\langle 1,-x z\rangle D(x)$. Also, $z \in D\langle 1, x\rangle$ implies $D(x z)=D(x)+D(z)$. Now

$$
\begin{aligned}
\langle 1,-x z\rangle D(x) & =x \cdot\langle x,-z\rangle D(x)=\langle x,-z\rangle D(x) \\
& =\langle 1,-x\rangle D(x)+\langle 1,-z\rangle D(x),
\end{aligned}
$$

since $\langle 1,1\rangle D(x)=0$. Working the same way with $\langle 1,-x g\rangle D(x)$ and $\langle 1,-x g\rangle D(z)$ yields:

$$
\begin{aligned}
\langle 1, & -x\rangle D(x)+\langle 1,-z\rangle D(x) \\
\quad & \langle 1,-x\rangle D(x)+\langle 1,-g\rangle D(x)+\langle 1,-x\rangle D(z)+\langle 1,-g\rangle D(z) .
\end{aligned}
$$

(*) $\langle 1,-z\rangle D(x)=\langle 1,-g\rangle D(x)+\langle 1,-x\rangle D(z)+\langle 1,-g\rangle D(z)$.

But $z \in D\langle 1, x\rangle$ implies $\langle 1,-z\rangle D(x)=\langle 1,-x\rangle D(z)$, and $z \in D\langle 1, g\rangle$ implies $\langle 1,-g\rangle D(z)=\langle 1,-z\rangle D(g)=0$. Then $(*)$ yields $\langle 1,-g\rangle D(x)=0$.

REMARK. Lemma 2.3 does not hold for Witt rings $R$ of local type and characteristic 2. For example, we may describe $L_{4,0}$ by taking $G=$ $(a, b, c, d)$-here $(S)$ denotes the group generated by $S$-and $D\langle 1, a\rangle=$ $(a, b, c), \quad D\langle 1, b\rangle=(a, b, c d), \quad D\langle 1, c\rangle=(a, c, b d)$ and $D\langle 1, d\rangle=$ $(a b, a c, d)$. The map given by $D(a)=\langle 1, a\rangle, D(b)=\langle 1, b\rangle, D(c)=$ $\langle 1, a\rangle$ and $D(d)=0$ induces a derivation. But $D(d)=0$ while $\langle 1,-d\rangle D(a) \neq 0$. 
Proposition 2.4. Let $R$ be $L_{2 n, 1}$ or $L_{2 n+1},(n \geq 1)$ and let $G$ be the associated group. Then $\operatorname{Der}(R)$ is generated by the derivations $d(H, \beta)(c f$. (1.3)). Here $H$ is a subgroup of index 2 in $G$ containing -1 and $\beta$ is a scalar multiple of a Pfister form with $H \subset x D(\beta)$, for some $x \in G$.

Proof. Let $0 \neq D \in \operatorname{Der}(R)$ and let $K=\operatorname{ker}\left(\left.D\right|_{G}\right)$. Suppose first that $i_{G}(K)=2$. Let $x \notin K$ and $\beta=D(x)$. Note that $\beta$ is a scalar multiple of a Pfister form by (1.1) and (2.2). By (2.3), if $k \in K$ then $\langle 1,-k\rangle D(x)=0$ so that $K$ is contained in a multiple of the value set of $\beta$. Also, $D(k)=0$ and $D(k x)=k D(x)=D(x)$. Thus $D=d(K, \beta)$.

Now suppose $i_{G}(K) \geq 4$. Fix $x \notin K$ and let $D(x)=\beta$. We will complete the proof by showing there exists $D^{\prime}$, a sum of $d(H, \alpha)$ 's, such that $\operatorname{ker}\left(\left.\left(D-D^{\prime}\right)\right|_{G}\right) \supset K \cup x K$.

By (2.2), $\beta=\rho,\langle 1,-y\rangle$ or $z\langle 1,-y\rangle$, where $y \in D\langle 1,1\rangle$ and $z \notin$ $D\langle 1,-y\rangle$. If $\beta=\rho$, write $G=\{1, x\} H$, with $K \subset H$ and $i_{G}(H)=2$. Then $K \cup x K \subset \operatorname{ker}\left(\left.(D-d(H, \rho))\right|_{G}\right)$. If $\beta=\langle 1,-y\rangle$ and $x \notin\langle 1,-y\rangle$ then (2.3) implies $K \subset D\langle 1,-y\rangle$ and so

$$
K \cup x K \subset \operatorname{ker}\left(\left.(D-d(D\langle 1,-y\rangle,\langle 1,-y\rangle))\right|_{G}\right) .
$$

Now suppose $\beta=\langle 1,-y\rangle$ and $x \in D\langle 1,-y\rangle$. Let $H$ be a subgroup of index 2 in $G$, containing $K$ but not $x$. Then $H=D\langle 1,-w\rangle$, for some $w \in G$, by (2.1). We have $x \notin D\langle 1,-w\rangle$ and so $x \notin D\langle 1,-w y\rangle$. Also $K \subset D\langle 1,-y\rangle \cap D\langle 1,-y w\rangle$ by (2.3). Set $D^{\prime}=d(D\langle 1,-w y\rangle$, $\langle 1,-w y\rangle)+d(H,-y\langle 1,-w\rangle)$. Then $D^{\prime}(x)=\langle 1,-w y\rangle \perp-y\langle 1,-w\rangle$ $=\langle 1,-y\rangle=\beta$ and $D^{\prime}(K)=0$. So $\operatorname{ker}\left(\left.\left(D-D^{\prime}\right)\right|_{G}\right) \supset K \cup x K$.

Lastly, suppose $\beta=z\langle 1,-y\rangle$. By the above argument, there exists $D^{\prime}$, a sum of $d(H, \alpha)$ 's, such that $K \cup x K \subset \operatorname{ker}\left(\left.\left(z D-D^{\prime}\right)\right|_{G}\right)$. But $z D^{\prime}$ is still a sum of $d(H, \alpha)$ 's and $K \cup x K \subset \operatorname{ker}\left(\left.\left(D-z D^{\prime}\right)\right|_{G}\right)$.

COROllary 2.5. Let $R$ be $L_{2 n, 1}$ or $L_{2 n+1},(n \geq 1)$ with associated group $G$. Then $\operatorname{Der}(R) \subset \operatorname{Hom}(G, R)$. That is, if $D \in \operatorname{Der}(R)$ and $x, y \in$ $G$ then $D(x y)=D(x)+D(y)$.

Proof. This holds for $D=d(H, \beta)$ and hence for all derivations by (2.4).

REMARKS. (1) (2.4) and (2.5) fail for Witt rings of local type and characteristic 2 . The example given after (2.3) has $d(a d)=d D(a) \neq D(a)$ $+D(d)$, contradicting (2.5) and hence (2.4). 
(2) There are relations among the $d(H, \beta)$ 's which are difficult to determine explicitly. We will compute $\operatorname{Der}\left(L_{2 n, 1}\right), \operatorname{Der}\left(L_{2 n+1}\right), n \geq 1$, by an inductive argument instead.

We note that $L_{1}=\mathbf{Z}$.

Lemma 2.6. Let $R$ be $L_{2 n, 1}$ or $L_{2 n+1},(n \geq 1)$ and let $G$ be the group associated to $R$. Let $S$ be the Witt ring of local type with group $K$ where $2|K|=|G|$ and $\operatorname{char}(S) \neq 2$ (i.e. $S$ is $L_{2 n-1}$ or $L_{2 n, 1}$ ). Fix $a \in G \backslash\{1\}$ with $-1 \notin D\langle 1, a\rangle$.

(1) $D\langle 1, a\rangle$ has the induced quaternionic structure and $S \cong$ $W(D\langle 1, a\rangle)$.

(2) There is a (group) isomorphism:

$$
\alpha: \operatorname{ann}_{S}\langle 1,1\rangle \rightarrow \operatorname{ann}_{R}\langle 1,1\rangle \cap \operatorname{ann}_{R}\langle 1, a\rangle
$$

with $\alpha(k q)=k \alpha(q)$ for all $q \in \operatorname{ann}_{S}\langle 1,1\rangle$ and $k \in K$ ( $K$ is identified with $D\langle 1, a\rangle)$.

Proof. There is an orthogonal decomposition $G=\{1,-a\} \perp D\langle 1, a\rangle$ and so $D\langle 1, a\rangle$ inherits a quaternionic structure with $a$ as the distinguished element (cf. [1]). The Witt ring of $D\langle 1, a\rangle$ clearly is of local type, $a \neq 1$ and $2|D\langle 1, a\rangle|=|G|$, so $S \cong W(D\langle 1, a\rangle)$. We now identify $S$ with $W(D\langle 1, a\rangle)$ and $K$ with $D\langle 1, a\rangle$.

Define $\alpha_{0}: I_{S} \rightarrow I_{R}$ by $\sum_{i}\left\langle y_{2 i-1}, y_{2 i}\right\rangle \mapsto \sum_{i}\left\langle y_{2 i-1},-a y_{2 i}\right\rangle$. To show $\alpha_{0}$ is well-defined, it suffices to check on binary forms, by Witt's theorem on chain equivalence [5, p. 31]. Suppose $\left\langle y_{1}, y_{2}\right\rangle=\left\langle x_{1}, x_{2}\right\rangle$ in $S$. Then $y_{1} y_{2}=x_{1} x_{2}$ and $x_{1} y_{1} \in D_{S}\left\langle 1, y_{1} y_{2}\right\rangle$. Since $x_{1}, y_{1} \in K=D_{R}\langle 1, a\rangle$ we have $x_{1}, y_{1} \in D_{R}\left\langle 1, y_{1} y_{2}\right\rangle \cap D_{R}\langle 1, a\rangle \subset D_{R}\left\langle 1,-a y_{1} y_{2}\right\rangle$. Thus $\left\langle y_{1},-a y_{2}\right\rangle=\left\langle x_{1},-a x_{2}\right\rangle$ in $R$. Note that $\alpha_{0}$ is clearly a (group) homomorphism and that $\alpha_{0}(k q)=k \alpha_{0}(q)$ for all $k \in K, q \in I_{S}$. Further,

$$
\langle 1, a\rangle \alpha_{0}\left(\sum_{i}\left\langle y_{2 l-1}, y_{2 l}\right\rangle\right)=\sum_{i} y_{2 l-1}\left\langle\left\langle a,-y_{2 i-1} y_{2 i}\right\rangle\right\rangle=0
$$

since $y_{2 l-1} y_{2 i} \in D\langle 1, a\rangle$. Hence $\alpha_{0}\left(I_{S}\right) \subset \operatorname{ann}_{R}\langle 1, a\rangle$.

Let $\alpha$ be the restriction of $\alpha_{0}$ to $\operatorname{ann}_{S}\langle 1,1\rangle$. Then $\alpha: \operatorname{ann}_{S}\langle 1,1\rangle \rightarrow$ $\operatorname{ann}_{R}\langle 1,1\rangle \cap \operatorname{ann}_{R}\langle 1, a\rangle$. Now $\left|\operatorname{ann}_{S}\langle 1,1\rangle\right|=|K|=\frac{1}{2}|G|$, by (2.2). Using (2.2) again shows $\operatorname{ann}_{R}\langle 1,1\rangle \cap \operatorname{ann}_{R}\langle 1, a\rangle$ consists of $0, \rho$ and pairs $\langle 1, x\rangle, x^{\prime}\langle 1, x\rangle$ where $-x \in D_{R}\langle 1,1\rangle \cap D_{R}\langle 1, a\rangle \backslash\{1\}, x^{\prime} \notin D\langle 1, x\rangle$. Thus

$$
\left|\operatorname{ann}_{R}\langle 1,1\rangle \cap \operatorname{ann}_{R}\langle 1, a\rangle\right|=2\left|D_{R}\langle 1,1\rangle \cap D_{R}\langle 1, a\rangle\right|=\frac{1}{2}|G| .
$$

To complete the proof then, we need only show $\alpha$ is injective. 
Suppose $y, y^{\prime} \in K$ with $\langle 1, y\rangle \in \operatorname{ann}_{S}\langle 1,1\rangle$ and $\alpha\left(y^{\prime}\langle 1, y\rangle\right)=0$. Then $\langle 1,-a y\rangle=0$ in $R, a=y$ and $\langle 1, y\rangle=0$ in $S$. Let $y, y^{\prime} \in K$, $q=\left\langle\left\langle y,-y^{\prime}\right\rangle\right\rangle$ and suppose $\alpha(q)=0$. Then $\langle 1,-a y\rangle \perp-y^{\prime}\langle 1,-a y\rangle$ $=0, y^{\prime} \in D_{R}\langle 1,-a y\rangle \cap D_{R}\langle 1, a\rangle \subset D_{R}\langle 1, y\rangle$. But then $y^{\prime} \in D_{S}\langle 1, y\rangle$ and $q=0$ in $S$. By (2.2), $\operatorname{ann}_{S}\langle 1,1\rangle$ consists of such $y^{\prime}\langle 1, y\rangle,\left\langle\left\langle y,-y^{\prime}\right\rangle\right\rangle$ and so $\alpha$ is injective.

Denote the inverse of $\alpha$ by $\beta$. Note that $\beta(k q)=k \beta(q)$ for all $k \in D_{R}\langle 1, a\rangle$ and $q \in \operatorname{ann}_{R}\langle 1,1\rangle \cap \operatorname{ann}_{R}\langle 1, a\rangle$. Note also under the identification of $K$ with $D\langle 1, a\rangle, S$ is a subring of $R$. We continue with the notations of (2.6).

Corollary 2.7. Let $A=\{D \in \operatorname{Der}(R) \mid D(a)=0\}$. Then $\beta_{*}: A \rightarrow$ $\operatorname{Der}(S)$ is a Lie algebra isomorphism, where $\beta_{*}(D)=\beta\left(\left.D\right|_{S}\right)$.

Proof. We first show $\beta_{*}$ is well-defined. If $x \in G$ and $D \in A$ then $\langle 1, a\rangle D(x)=0$ by (2.3). Thus $D(R) \subset \operatorname{ann}_{R}\langle 1,1\rangle \cap \operatorname{ann}_{R}\langle 1, a\rangle$, the domain of $\beta$. Further, if $q_{1}, q_{2} \in S$ then

$$
\begin{aligned}
\beta_{*}(D)\left(q_{1} q_{2}\right) & =\beta\left(D\left(q_{1} q_{2}\right)\right)=\beta\left(q_{1} D\left(q_{2}\right)+q_{2} D\left(q_{1}\right)\right) \\
& =q_{1} \beta_{*}(D)\left(q_{2}\right)+q_{2} \beta_{*}(D)\left(q_{1}\right),
\end{aligned}
$$

since $\beta(k q)=k \beta(q)$ for any $k \in K, q \in \operatorname{ann}_{R}\langle 1,1\rangle \cap \operatorname{ann}_{R}\langle 1, a\rangle$. Thus $\beta_{*}(D) \in \operatorname{Der}(S)$ for all $D \in A$.

Now $\beta_{*}$ is additive and if $D_{1}, D_{2} \in A$ and $k \in K$ write $D_{2}(k)=$ $\sum_{i}\left\langle y_{2 i-1}, y_{2 i}\right\rangle \in S$. Then:

$$
\begin{aligned}
\beta_{*}\left(D_{1}\right) \circ \beta_{*}\left(D_{2}\right) & (k)=\beta_{*}\left(D_{1}\right)\left(\beta\left(\sum_{i}\left\langle y_{2 i-1}, y_{2 i}\right\rangle\right)\right) \\
= & \beta_{*}\left(D_{1}\right)\left(\sum_{i}\left\langle y_{2 i-1},-a y_{2 i}\right\rangle\right)=\beta\left(\sum D_{1}\left\langle y_{2 i-1},-a y_{2 i}\right\rangle\right) \\
= & \beta\left(\sum D_{1}\left\langle y_{2 i-1}, y_{2 i}\right\rangle\right)
\end{aligned}
$$

since $D_{1}\left(a y_{2 i}\right)=a D_{1}\left(y_{2 i}\right)=D_{1}\left(y_{2 i}\right)$ by $(2.3)$. Thus $\beta_{*}\left(D_{1}\right) \circ \beta_{*}\left(D_{2}\right)=$ $\beta_{*}\left(D_{1} \circ D_{2}\right)$. In particular, $\beta_{*}\left(\left[D_{1}, D_{2}\right]\right)=\left[\beta_{*} D_{1}, \beta_{*} D_{2}\right]$ and $\beta_{*}$ is a Lie algebra homomorphism.

If, for $D \in A, \beta_{*}(D)=0$ then $\left.D\right|_{K}=0$, since $\beta$ is an isomorphism. Hence $G=\{1,-a\} K \subset \operatorname{ker}\left(\left.D\right|_{G}\right)$ and $D=0$. Thus $\beta_{*}$ is injective. To show $\beta_{*}$ is surjective it suffices to show that the generators $d(H, \alpha)$ of $\operatorname{Der}(S)$ (2.4) are in the image of $\beta$. Let $H$ be a subgroup of index 2 in $K$ containing $a$. By $(2.1), H=D_{S}\langle 1, x\rangle$. Let $\alpha$ be $\langle 1, x\rangle, x^{\prime}\langle 1, x\rangle$ or $\rho$, 
where $x^{\prime} \notin D_{S}\langle 1, x\rangle$ and $\rho=\left\langle\left\langle x, a x^{\prime}\right\rangle\right\rangle$. We need to show $d(H, \alpha)$ is in the image of $\beta_{*}$. We check this only for $\alpha=\langle 1, x\rangle$, the other cases being similar.

Set $H^{\prime}=D_{R}\langle 1,-a x\rangle$. Note that $H\{1,-1\} \subset H^{\prime}$, since $H \subset$ $D_{R}\langle 1, a\rangle \cap D_{R}\langle 1, x\rangle$ and $x \in K=D_{R}\langle 1, a\rangle, a \in H \subset D_{R}\langle 1, x\rangle$ implies $-1 \in D_{R}\langle 1,-a x\rangle$. Then $\beta_{*}\left(d\left(H^{\prime},\langle 1,-a x\rangle\right)=d(H,\langle 1, x\rangle)\right.$.

THEOREM 2.8. Let $R$ be $L_{2 n, 1}$ or $L_{2 n+1}$ with $n \geq 1$, and let $G$ be the associated group. Let $a \in G$ with $-a \notin D\langle 1,1\rangle$. Let $A=\{D \in$ $\operatorname{Der}(R) \mid D(a)=0\}$. Let $S=L_{2 n-1}$ if $R=L_{2 n, 1}$ and $S=L_{2 n, 1}$ if $R=$ $L_{2 n+1}$.

(1) There is an exact sequence of groups:

$$
0 \rightarrow A \rightarrow \operatorname{Der}(R) \stackrel{e}{\rightarrow} \operatorname{ann}_{R}\langle 1,1\rangle \rightarrow 0
$$

where $e(D)=D(a)$ and $A$ is Lie isomorphic to $\operatorname{Der}(S)$.

(2) $\operatorname{dim} \operatorname{Der}(R)=\frac{1}{2}(n+2)(n-1)$.

Proof. (1) We need only show $e$ is surjective by (2.7). Let $\alpha \in$ $\operatorname{ann}_{R}\langle 1,1\rangle$. If $\alpha=\rho,\langle 1,-x\rangle$ or $x^{\prime}\langle 1,-x\rangle$ where $x^{\prime} \notin D_{R}\langle 1,-x\rangle$ and $a \notin D_{R}\langle 1,-x\rangle$ then $e(d(D\langle 1,-x\rangle, \alpha))=\alpha$. By (2.2) we may thus assume $\alpha=\langle 1,-x\rangle$ with $a \in D_{R}\langle 1,-x\rangle$. Choose $z$ such that $a \notin$ $D_{R}\langle 1,-z\rangle$. Then

$$
\begin{aligned}
e(d(D\langle 1,-x z\rangle,\langle 1,-x z\rangle)-x d(D\langle 1,-z\rangle,\langle 1,-z\rangle)) & \\
& =\langle 1,-x z\rangle+-x\langle 1,-z\rangle=\alpha .
\end{aligned}
$$

(2) $\operatorname{dim}(\operatorname{Der}(R))=\operatorname{dim}(\operatorname{Der}(S))+n$, by (1) and (2.2). Since $\operatorname{Der}\left(L_{1}\right)$ $=\operatorname{Der}(\mathbf{Z})=0$ we have

$$
\operatorname{dim}(\operatorname{Der}(R))=n+(n-1)+\cdots+2=\frac{1}{2}(n+2)(n-1) .
$$

We turn now to the Witt rings $L_{2 n, 0}$ with $n \geq 2$. Let $G$ be the group associated to $L_{2 n, 0}$ and let $q: G \times G \rightarrow \mathbf{Z}_{2}$ be the associated linked quaternionic map. We require the following construction:

Fix $a, b \in G$ with $a \notin D\langle 1, b\rangle$. There is an orthogonal sum $G=$ $\{1, a, b, a b\} \perp D\langle 1, a\rangle \cap D\langle 1, b\rangle$. Set $H=\{1, a, b, a b\}$ and $K=$ $D\langle 1, a\rangle \cap D\langle 1, b\rangle$. By [1] $G$ induces a quaternionic structure on $H$ and $K$. In particular, for $k \in K$ we write $D_{K}\langle 1, k\rangle=D_{R}\langle 1, k\rangle \cap K$.

Embed $K$ into a group $\bar{K}$ with $|\bar{K}|=2|K|$; say $\bar{K}=\{1, c\} K$. For $k \in K \backslash\{1\}$ define:

$$
\begin{aligned}
& D_{\bar{K}}\langle 1, k\rangle=\left\{1, c k^{\prime}\right\} D_{K}\langle 1, k\rangle, \quad \text { where } k^{\prime} \in K \backslash D_{K}\langle 1, k\rangle, \\
& D_{\bar{K}}\langle 1, c k\rangle=\{1, c\} D_{K}\langle 1, k\rangle .
\end{aligned}
$$


We also set $D_{\bar{K}}\langle 1,1\rangle=K$ and $D_{\bar{K}}\langle 1, c\rangle=\bar{K}$. We further define $\bar{q}$ : $\bar{K} \times \bar{K} \rightarrow \mathbf{Z}_{2}$ by $\bar{q}(x, y)=0$ iff $y \in D_{\bar{K}}\langle 1, c x\rangle$. (We are thus taking $c$ to be the distinguished element of $\bar{K}$.)

LEMMA 2.9. (1) $\bar{q}$ is a linked quaternionic mapping.

(2) $W \bar{K} \cong L_{2 n-1}$.

Proof. (1) Note that $x \in D_{\bar{K}}\langle 1, x\rangle$ for all $x \in \bar{K}$. Suppose $x \in$ $D_{\bar{K}}\langle 1, y\rangle$. We will show $c y \in D_{\bar{K}}\langle 1, c x\rangle$ for the case $y \in K$ (the case $y \in c K$ is similar). If $x \in D_{K}\langle 1, y\rangle$ then $x \in D_{R}\langle 1, y\rangle, y \in D_{R}\langle 1, x\rangle$ since $-1=1$ in $G$, and so $y \in D_{K}\langle 1, x\rangle$. Hence $c y \in D_{\bar{K}}\langle 1, c x\rangle$. If $x=c y^{\prime} x^{\prime}$ with $y^{\prime} \in K \backslash D_{K}\langle 1, y\rangle$ and $x^{\prime} \in D_{K}\langle 1, y\rangle$ then $y \in$ $K \backslash D_{K}\left\langle 1, x^{\prime} y^{\prime}\right\rangle$. So $c y \in D_{\bar{K}}\left\langle 1, x^{\prime} y^{\prime}\right\rangle=D_{K}\langle 1, c x\rangle$.

We lastly check $\bar{q}$ is linked. Suppose $\bar{q}\left(x_{1}, y_{1}\right)=\bar{q}\left(x_{2}, y_{2}\right)$, with $x_{i}, y_{j} \in \bar{K},(i, j=1,2)$. If $\bar{q}\left(x_{i}, y_{i}\right)=0, i=1,2$, then $\bar{q}\left(x_{1}, y_{1}\right)=\bar{q}\left(x_{1}, 1\right)$ $=\bar{q}\left(x_{2}, 1\right)=\bar{q}\left(x_{2}, y_{2}\right)$. If $\bar{q}\left(x_{i}, y_{i}\right) \neq 0$, for $i=1,2$, then $y_{1} \neq 1$ and $y_{2} \neq 1$. Since $\bar{K}$ cannot be written as the union of two proper subgroups, there exists $w \notin D_{\bar{K}}\left\langle 1, c x_{1}\right\rangle \cup D_{\bar{K}}\left\langle 1, c x_{2}\right\rangle$. Then $\bar{q}\left(x_{1}, y_{1}\right)=\bar{q}\left(\bar{x}_{1}, w\right)=$ $\bar{q}\left(x_{2}, w\right)=\bar{q}\left(x_{2}, y_{2}\right)=1$.

(2) $\bar{q}$ has a range of two elements, so the Witt ring $W \bar{K}$ it induces is of local type. Since the distinguished element $c$ is not 1 and $|\bar{K}|=2|K|$ $=\frac{1}{2}|G|, W \bar{K} \cong L_{2 n-1}$.

We continue with all of the notations introduced before (2.9) but now writing $\bar{R}$ for $W \bar{K} \cong L_{2 n-1}$.

LEMMA 2.10. There is an additive group isomorphism

$$
\alpha: \operatorname{ann}_{\bar{R}}\langle 1,1\rangle \rightarrow \operatorname{ann}_{R}\langle 1, a\rangle \cap \operatorname{ann}_{R}\langle 1, b\rangle
$$

such that $\alpha(y q)=y \alpha(q)$ for all $y \in \bar{K}, q \in \operatorname{ann}_{\bar{R}}\langle 1,1\rangle$.

Proof. Since $D_{\bar{K}}\langle 1,1\rangle=K$, a form in $\operatorname{ann}_{\bar{R}}\langle 1,1\rangle$ looks like $\sum_{i}\left\langle y_{2 i-1}, c y_{2 i}\right\rangle$, with each $y_{j} \in K(2.2)$. Define $\alpha$ by:

$$
\alpha\left(\sum_{i}\left\langle y_{2 i-1}, c y_{2 i}\right\rangle\right)=\sum_{i}\left\langle y_{2 i-1}, y_{2 i}\right\rangle \text {. }
$$

To show $\alpha$ is well-defined it suffices to check on binary forms. Suppose $\left\langle y_{1}, c y_{2}\right\rangle=\left\langle y_{3}, c y_{4}\right\rangle$ in $\bar{R}$. Then $y_{1} y_{2}=y_{3} y_{4}$ and $y_{1} y_{3} \in D_{\bar{K}}\left\langle 1, c y_{1} y_{2}\right\rangle=$ $\{1, c\} D_{K}\left\langle 1, y_{1} y_{2}\right\rangle$. Since $y_{1} y_{3} \in K$ we get $y_{1} y_{3} \in D_{K}\left\langle 1, y_{1} y_{2}\right\rangle \subset$ $D_{R}\left\langle 1, y_{1} y_{2}\right\rangle$, so $\left\langle y_{1}, y_{2}\right\rangle=\left\langle y_{3}, y_{4}\right\rangle$ in $R$. Also, since each $y_{j} \in K=$ $D\langle 1, a\rangle \cap D\langle 1, b\rangle,\langle 1, a\rangle \cdot\left\langle y_{2 i-1}, y_{2 i}\right\rangle=0=\operatorname{ann}_{R}\langle 1, a\rangle \cap \operatorname{ann}_{R}\langle 1, b\rangle$. 
Now $\alpha$ is clearly a homomorphism and $\alpha(y q)=y \alpha(q)$ for all $y \in \bar{K}$ and $q \in \operatorname{ann}_{\bar{R}}\langle 1,1\rangle$. To show $\alpha$ is an isomorphism we first

Claim. $\operatorname{ann}_{R}\langle 1, a\rangle \cap \operatorname{ann}_{R}\langle 1, b\rangle=\{0, \rho\} \cup\left\{\langle 1, k\rangle, k^{\prime}\langle 1, k\rangle \mid k \in K\right.$ and $\left.k^{\prime} \in K \backslash D_{R}\langle 1, k\rangle\right\}$.

Let $S$ be the set on the right-hand side. Since $K=D\langle 1, a\rangle \cap D\langle 1, b\rangle$ and $-1=1$ in $G$ we have $S \subset \operatorname{ann}_{R}\langle 1, a\rangle \cap \operatorname{ann}_{R}\langle 1, b\rangle$. Let $q \in$ $\operatorname{ann}_{R}\langle 1, a\rangle \cap \operatorname{ann}_{R}\langle 1, b\rangle$. If $q$ is not a binary form then $q=0$ or $\rho$, since $q \in I_{R}$. So suppose $q=y \cdot\langle 1, x\rangle$, with $x, y \in G$. Then $\langle 1, a\rangle \cdot\langle 1, x\rangle=$ $0=\langle 1, b\rangle \cdot\langle 1, x\rangle$ implies $x \in K$. If $y \in D\langle 1, x\rangle$ then $y\langle 1, x\rangle=\langle 1, x\rangle$ $\in S$. Suppose then that $y \notin D\langle 1, x\rangle$. Now $K$ is a subgroup of index 4 in $G$. $K$ is then contained in precisely three subgroups of index 2, namely $D\langle 1, x\rangle, D\langle 1, b\rangle$ and $D\langle 1, a b\rangle$. If $K \subset D\langle 1, x\rangle$ then $x \in\{a, b, a b\}=$ $H \backslash\{1\}$, which is impossible since $x \in K$ and $K \cap H=\{1\}$. Thus $K \not \subset$ $D\langle 1, x\rangle$. Choose $k^{\prime} \in K \backslash D\langle 1, x\rangle$. Then $q=y\langle 1, x\rangle=k^{\prime}\langle 1, x\rangle \in S$. This proves the claim.

The claim quickly yields that $\alpha$ is surjective since fror $k, k^{\prime} \in K$, $\alpha\left(\left\langle k^{\prime}, k^{\prime} k c\right\rangle\right)=k^{\prime}\langle 1, k\rangle$, and if $k^{\prime} \notin D\langle 1, k\rangle$ then $\alpha\left(\langle 1, c k\rangle+k^{\prime}\langle 1, c k\rangle\right)$ $=\left\langle\left\langle k, k^{\prime}\right\rangle\right\rangle=\rho$. Further the claim shows $\left|\operatorname{ann}_{R}\langle 1, a\rangle \cap \operatorname{ann}_{R}\langle 1, b\rangle\right|=$ $2|K|=|\bar{K}|$. By (2.2), $\left|\operatorname{ann}_{\bar{R}}\langle 1,1\rangle\right|=|\bar{K}|$ also. Thus $\alpha$ is an isomorphism.

LEMMA 2.11. Let $R=L_{2 n, 0}$ and $\bar{R}=W \bar{K} \cong L_{2 n-1}(n \geq 2)$ as before. Let $A=\{D \in \operatorname{Der}(R) \mid D(a)=D(b)=0\}$. Then $A$ is Lie isomorphic to $\operatorname{Der}(\bar{R})$.

Proof. We first show that for $D \in A, \quad D(R) \subset \operatorname{ann}_{R}\langle 1, a\rangle \cap$ $\operatorname{ann}_{R}\langle 1, b\rangle$. For $g \in G$, if $g \in D\langle 1, a\rangle$ then $\langle 1, a\rangle D(g)=\langle 1, g\rangle D(a)=$ 0 . If $g \notin D\langle 1, a\rangle$ then $b g \in D\langle 1, a\rangle$, since $b \notin D\langle 1, a\rangle$. Hence $\langle 1, a\rangle D(b g)=\langle 1, b g\rangle D(a)=0$. So $0=b\langle 1, a\rangle D(g)+g\langle 1, a\rangle D(b)$. Since $D(b)=0$ also $\langle 1, a\rangle D(g)=0$. Thus in each case $D(g) \in$ $\operatorname{ann}_{R}\langle 1, a\rangle$. Similarly, $D(g) \in \operatorname{ann}_{R}\langle 1, b\rangle$.

For $D \in A$ define $\bar{D}: \bar{K} \rightarrow \operatorname{ann}_{R}\langle 1, a\rangle \cap \operatorname{ann}_{R}\langle 1, b\rangle$, by $\bar{D}(k)=$ $\bar{D}(c k)=D(k)$, for all $k \in K$. Let $\beta=\alpha^{-1}$, where $\alpha$ is the isomorphism of (2.10). Note $\beta(k q)=k \beta(q)$ for all $k \in K, q \in \operatorname{ann}_{R}\langle 1, a\rangle \cap$ $\operatorname{ann}_{R}\langle 1, b\rangle$. Define $\beta_{*}: A \rightarrow \operatorname{Der}(\bar{R})$ by:

$$
\beta_{*}(D)=\beta \circ \bar{D} \text {. }
$$

Note that we have already shown the image of $\bar{D}$ is in the domain of $\beta$.

We next show $\beta_{*}(D) \in \operatorname{Der}(\bar{R})$. First, $\beta_{*}(D)(c)=\beta_{*}(\bar{D}(c))=$ $\beta(D(1))=0$. Let $x_{1}, x_{2} \in \bar{K}$ and write $x_{i}=c^{\varepsilon_{l}} y_{i}$, where $\varepsilon_{\imath} \in\{0,1\}$ and 
$y_{i} \in K$. Then

$$
\begin{gathered}
\beta_{*}(D)\left(x_{1} x_{2}\right)=\beta\left(D\left(y_{1} y_{2}\right)\right)=\beta\left(y_{1} D\left(y_{2}\right)+y_{2} D\left(y_{1}\right)\right) \\
=y_{1} \beta\left(D\left(y_{2}\right)\right)+y_{2} \beta\left(D\left(y_{1}\right)\right)=y_{1} \beta_{*}(D)\left(x_{2}\right)+y_{2} \beta_{*}(D)\left(x_{1}\right) \\
=x_{1} \beta_{*}(D)\left(x_{2}\right)+x_{2} \beta_{*}(D)\left(x_{1}\right),
\end{gathered}
$$

since $\langle 1,1\rangle \beta(D(z))=0$ in $\bar{R}$, for any $z \in \bar{K}$, implies $c \beta(D(z))=$ $\beta(D(z))$.

Next suppose $x_{1} \in D_{\bar{R}}\left\langle 1, x_{2}\right\rangle$. Then, by our construction, $y_{1} \in$ $D_{K}\left\langle 1, y_{2}\right\rangle$ unless $x_{1}=c y_{1}, x_{2}=y_{2}$. If $y_{1} \in D_{K}\left\langle 1, y_{2}\right\rangle$ then $D\left(y_{1} y_{2}\right)=$ $D\left(y_{1}\right)+D\left(y_{2}\right)$ and so $\beta_{*}(D)\left(x_{1} x_{2}\right)=\beta_{*}(D)\left(x_{1}\right)+\beta_{*}(D)\left(x_{2}\right)$. So suppose $y_{1}, y_{2} \in K$ with $c y_{1} \in D_{\bar{R}}\left\langle 1, y_{2}\right\rangle$ and $y_{1} \notin D_{\bar{R}}\left\langle 1, y_{2}\right\rangle$. Then $y_{1} \notin$ $D_{R}\left\langle 1, b y_{2}\right\rangle$, since $y_{1} \in K \subset D_{R}\langle 1, b\rangle$. Also, $a \in D_{R}\left\langle 1, y_{2}\right\rangle, a \notin D_{R}\langle 1, b\rangle$ imply $a \notin D_{R}\left\langle 1, b y_{2}\right\rangle$. Thus $a y_{1} \in D_{R}\left\langle 1, b y_{2}\right\rangle$ and $D\left(a b y_{1} y_{2}\right)=D\left(a y_{1}\right)+$ $D\left(b y_{2}\right)$. Now $D(a b)=0$ implies $D\left(a b y_{1} y_{2}\right)=a b D\left(y_{1} y_{2}\right)$. And $a b D\left(y_{1} y_{2}\right)$ $=D\left(y_{1} y_{2}\right)$ since $D(R) \subset \operatorname{ann}_{R}\langle 1, a\rangle \cap \operatorname{ann}_{R}\langle 1, b\rangle$. Thus $D\left(a b y_{1} y_{2}\right)=$ $D\left(y_{1} y_{2}\right)$ and similarly $D\left(a y_{1}\right)=D\left(y_{1}\right)$ and $D\left(b y_{2}\right)=D\left(y_{2}\right)$. We thus have $D\left(y_{1} y_{2}\right)=D\left(y_{1}\right)+D\left(y_{2}\right)$ and $\beta_{*}(D)\left(x_{1} x_{2}\right)=\beta_{*}(D)\left(x_{1}\right)+$ $\beta_{*}(D)\left(x_{2}\right)$. By (1.2) this completes the proof that $\beta_{*}(D) \in \operatorname{Der}(\bar{R})$.

Clearly $\beta_{*}$ is an additive map. Let $D_{1}, D_{2} \in A$ and $x=c^{\varepsilon} y \in \bar{K}$, with $\varepsilon \in\{0,1\}$ and $y \in K$. Let $D_{2}(y)=\sum_{i}\left\langle z_{2 i-1}, z_{2 i}\right\rangle$, with all $z_{j} \in K$. Then

$$
\begin{aligned}
\beta_{*}\left(D_{1}\right) \circ \beta_{*}\left(D_{2}\right)(x) & =\beta_{*}\left(D_{1}\right)\left(\beta\left(\sum_{i}\left\langle z_{2 i-1}, z_{2 i}\right\rangle\right)\right) \\
& =\beta_{*}\left(D_{1}\right)\left(\sum_{i}\left\langle z_{2 i-1}, c z_{2 i}\right\rangle\right)=\beta\left(D_{1}\left(\sum\left\langle z_{2 l-1}, z_{2 i}\right\rangle\right)\right) \\
& =\beta_{*}\left(D_{1} \circ D_{2}\right)(x) .
\end{aligned}
$$

Hence $\beta_{*}\left[D_{1}, D_{2}\right]=\left[\beta_{*} D_{1}, \beta_{*} D_{2}\right]$.

Since $\beta$ is an isomorphism, $\beta_{*}$ is clearly injective. To show $\beta_{*}$ is surjective, it suffices to show the generators of $\operatorname{Der}(\bar{R})$ given in (2.4) are in the image of $\beta_{*}$. A generator is $d(L, \gamma)$, where $\gamma \in \bar{R}$ and $L$ is a subgroup of index 2 in $\bar{K}$ containing $c$ and contained in a scalar multiple of $D_{\bar{R}}(\gamma)$. By (2.1) $L=D_{\bar{R}}\langle 1, x\rangle$, for some $x \in \bar{K}$ and $c \in D_{\bar{K}}\langle 1, x\rangle$, hence $c x \in D_{\bar{R}}\langle 1,1\rangle=K$. Then $\gamma$ is either $\langle 1, x\rangle, x^{\prime}\langle 1, x\rangle$ where $x^{\prime} \notin$ $D\langle 1, x\rangle$, or $\rho$ the unique non-trivial 2-fold Pfister form in $\bar{R}(2.2)$.

We will show $d(L, \gamma)$ with $\gamma=\langle 1, c y\rangle, y \in K$ and $L=D_{\bar{R}}\langle 1, c y\rangle$ is in the image of $\beta_{*}$. The other cases are similar. Let $D=d(D\langle 1, y\rangle$, $\langle 1, y\rangle) \in \operatorname{Der}(r)$. Since $y \in D\langle 1, a\rangle \cap D\langle 1, b\rangle, a, b \in D\langle 1, y\rangle$ and so 
$D \in A$. For $\varepsilon \in\{0,1\}$ and $x \in K$ we have $\beta_{*}(D)\left(c^{\varepsilon} x\right)=\beta(D(x))$, which is 0 if $x \in D_{R}\langle 1, y\rangle \cap K$, and is $\beta(\langle 1, y\rangle)=\gamma$ if $x \notin D_{R}\langle 1, y\rangle$. Thus the image $\beta_{*}(D)(\bar{K})$ is $\{0, \gamma\}$ and the kernel (in $\bar{K}$ ) is $\{1, c\} D_{K}\langle 1, y\rangle=$ $D_{\bar{R}}\langle 1, c y\rangle$. Hence $\beta_{*}(D)=d(L, \gamma)$.

LEMMA 2.12. Let $G$ be the group associated to $R=L_{2 n, 0}$ where $n \geq 1$. Then there exist subgroups $G_{1}, G_{2}$ of $G$ such that:

(1) $G=G_{1} \times G_{2}$

(2) $x_{1} \in D_{R}\left\langle 1, x_{2}\right\rangle$, for all $x_{1}, x_{2} \in G_{1}$;

(3) $y_{1} \in D_{R}\left\langle 1, y_{2}\right\rangle$, for all $y_{1}, y_{2} \in G_{2}$.

Further, if we fix $x_{0}, y_{0} \in G$ with $x_{0} \notin D_{R}\left\langle 1, y_{0}\right\rangle$ then we may assume $x_{0} \in G_{1}$ and $y_{0} \in G_{2}$.

Proof. If $n=1$ then $G=\left\{1, x_{0}, y_{0}, x_{0} y_{0}\right\}$ and the result is clear. Let $n \geq 2$ and fix $x_{0}, y_{0} \in G$ with $x_{0} \notin D_{R}\left\langle 1, y_{0}\right\rangle$. There is an orthogonal decomposition $G=\left\{1, x_{0}, y_{0}, x_{0} y_{0}\right\} \perp D_{R}\left\langle 1, x_{0}\right\rangle \cap D_{R}\left\langle 1, y_{0}\right\rangle$. Here $K=$ $D_{R}\left\langle 1, x_{0}\right\rangle \cap D_{R}\left\langle 1, y_{0}\right\rangle$ inherits a quaternionic structure and its Witt ring is $L_{2 n-2,0}$. By induction there exist $K_{1}, K_{2} \subset K$ satisfying conditions (1)-(3). Set $G_{1}=\left\{1, x_{0}\right\} K_{1}$ and $G_{2}=\left\{1, y_{0}\right\} K_{2}$. Then $G=G_{1} \times G_{2}$.

We check condition (2) (the proof of (3) is similar). Let $x_{1}, x_{2} \in G_{1}$ and write $x_{i}=x_{0}^{\varepsilon_{i}} x_{i}^{\prime}$ where, for $i=1,2, \varepsilon_{i} \in\{0,1\}$ and $x_{i}^{\prime} \in K_{1}$. Then $x_{1}^{\prime} \in D_{K}\left\langle 1, x_{2}^{\prime}\right\rangle$ and so $x_{1}^{\prime} \in D_{R}\left\langle 1, x_{2}^{\prime}\right\rangle$. Since $x_{1}^{\prime}, x_{2}^{\prime} \in K \subset D\left\langle 1, x_{0}\right\rangle$ we also have $x_{1} \in D_{R}\left\langle 1, x_{2}\right\rangle$ as desired.

THEOREM 2.13. Let $R=L_{2 n, 0}$ with $n \geq 2$ and let $G$ be the associated group. Fix $a, b \in G$ with $a \notin D_{R}\langle 1, b\rangle$. Let $A=\{D \in \operatorname{Der}(R) \mid D(a)=$ $D(b)=0\}$.

(1) There is an exact sequence of groups

$$
0 \rightarrow A \rightarrow \operatorname{Der}(R) \stackrel{e}{\rightarrow} I_{R} \times I_{R} \rightarrow 0
$$

where $e(D)=(D(a), D(b))$ and $A$ is a subalgebra Lie isomorphic to $\operatorname{Der}\left(L_{2 n-1}\right)$.

(2) $\operatorname{dim}(\operatorname{Der}(R))=(2 n+2)(2 n+1) / 2$.

Proof. (1) By (2.11) we need only show $e$ is surjective. By symmetry it is enough to show $0 \times I_{R} \subset \operatorname{im}(e)$. Then, since $e$ is additive and $x D \in$ $\operatorname{Der}(R)$ for all $x \in G, D \in \operatorname{Der}(R)$, it suffices to show $(0,\langle 1, g\rangle) \in \operatorname{im}(e)$ for all $g \in G$. 
If $g \in D\langle 1, a\rangle \backslash D\langle 1, b\rangle$ then $e(d(D\langle 1, g\rangle,\langle 1, g\rangle))=(0,\langle 1, g\rangle)$, where $d(D\langle 1, g\rangle,\langle 1, g\rangle)$ is the derivation of (1.3). If $g \in D\langle 1, a\rangle \cap$ $D\langle 1, b\rangle$ then $a g \in D\langle 1, a\rangle \backslash D\langle 1, b\rangle$ and

$$
\begin{aligned}
e(d(D\langle 1, a\rangle & ,\langle 1, a\rangle)+d(D\langle 1, a g\rangle,\langle 1, a g\rangle)) \\
& =(0,\langle 1, a\rangle)+(0,\langle 1, a g\rangle)=(0,\langle a, a g\rangle)=(0,\langle 1, g\rangle),
\end{aligned}
$$

since $a \in D\langle 1, g\rangle$. Thus $(0,\langle 1, g\rangle) \in \operatorname{im}(e)$ for all $g \in D\langle 1, a\rangle$. Since $D\langle 1, a\rangle$ has index 2 in $G$ and $b \notin D\langle 1, a\rangle$ it suffices to show (0, $\langle 1, b\rangle) \in \operatorname{im}(e)$.

Write $G=G_{1} \times G_{2}$ with subgroups $G_{1}, G_{2}$ satisfying (2.12) and with $a \in G_{1}, b \in G_{2}$. Define $D: G \rightarrow I_{R}$ by $D(x y)=x\langle 1, y\rangle$, for all $x \in G_{1}$, $y \in G_{2}$. Note that $D(a)=0$ and $D(b)=\langle 1, b\rangle$. We will be done if we show $D$ is a derivation.

We check the conditions of (1.2). Clearly $D(1)=0$. If $x_{1}, x_{2} \in G_{1}$ and $y_{1}, y_{2} \in G_{2}$ then

$$
\begin{aligned}
x_{1} y_{1} D\left(x_{2} y_{2} S\right)+x_{2} y_{2} D\left(x_{1} y_{1}\right) & =x_{1} x_{2} y_{1}\left\langle 1, y_{2}\right\rangle+x_{1} x_{2} y_{2}\left\langle 1, y_{1}\right\rangle \\
& =x_{1} x_{2}\left\langle y_{1}, y_{2}, y_{1} y_{2}, y_{1} y_{2}\right\rangle=x_{1} x_{2}\left\langle 1, y_{1} y_{2}\right\rangle,
\end{aligned}
$$

since $\langle 1,1\rangle=0$ in $I_{R}$ and $y_{1} \in D\left\langle 1, y_{1} y_{2}\right\rangle$. Thus $D\left(x_{1} y_{1} x_{2} y_{2}\right)=$ $x_{1} y_{1} D\left(x_{2} y_{2}\right)+x_{2} y_{2} D\left(x_{1} y_{1}\right)$. Now suppose $x_{1} y_{1} \in D\left\langle 1, x_{2} y_{2}\right\rangle$. We have:

$$
\begin{gathered}
D\left(x_{1} y_{1} x_{2} y_{2}\right)=x_{1} x_{2}\left\langle 1, y_{1} y_{2}\right\rangle \\
D\left(x_{1} y_{1}\right)+D\left(x_{2} y_{2}\right)=x_{1} x_{2}\left(x_{2}\left\langle 1, y_{1}\right\rangle+x_{1}\left\langle 1, y_{2}\right\rangle\right) .
\end{gathered}
$$

There are two cases. If $x_{1} \in D\left\langle 1, y_{2}\right\rangle$ then since $x_{1} \in D\left\langle 1, x_{2}\right\rangle$ we have $y_{1} \in D\left\langle 1, x_{2} y_{2}\right\rangle$. So $x_{1} y_{1} \in D\left\langle 1, x_{2} y_{2}\right\rangle$ implies $y_{1} \in D\left\langle 1, x_{2} y_{2}\right\rangle \cap$ $D\left\langle 1, y_{2}\right\rangle \subset D\left\langle 1, x_{2}\right\rangle$, and $x_{2} \in D\left\langle 1, y_{1}\right\rangle$. Using (*) gives $D\left(x_{1} y_{1}\right)+$ $D\left(x_{2} y_{2}\right)=x_{1} x_{2}\left\langle 1,1, y_{1}, y_{2}\right\rangle=D\left(x_{1} y_{1} x_{2} y_{2}\right)$.

Lastly, if $x_{1} \notin D\left\langle 1, y_{2}\right\rangle$ then, arguing as above, we obtain $x_{2} \notin$ $D\left\langle 1, y_{1}\right\rangle$. Choose $z \notin D\left\langle 1, y_{1}\right\rangle \cup D\left\langle 1, y_{2}\right\rangle$, which is possible since $G$ cannot be the union of two proper subgroups. Since $D\langle 1, x\rangle$ has index 2 in $G$ we have $y_{1} y_{2} \in D\langle 1, z\rangle$ and $z=D\left\langle 1, y_{1} y_{2}\right\rangle$. Then $x_{2}\left\langle 1, y_{1}\right\rangle=$ $z\left\langle 1, y_{1}\right\rangle$, and $x_{1}\left\langle 1, y_{2}\right\rangle=z\left\langle 1, y_{2}\right\rangle$. Using $\left.(*)\right\}$ gives

$$
D\left(x_{1} y_{1}\right)+D\left(x_{2} y_{2}\right)=x_{1} x_{2}\left\langle y_{1}, y_{2}, z, z\right\rangle=D\left(x_{1} y_{1} x_{2} y_{2}\right) \text {. }
$$

(2) We apply (2.8):

$$
\begin{aligned}
\operatorname{dim} \operatorname{Der}(R) & =\operatorname{dim} \operatorname{Der}\left(L_{2 n-1}\right)+2 \operatorname{dim} I_{R} \\
& =\frac{1}{2}(2 n+1)(2 n-2)+2(2 n+1)=\frac{1}{2}(2 n+2)(2 n+1) .
\end{aligned}
$$


3. Examples. Sections 1 and 2 can be used to compute the derivation algebra for any Witt ring of elementary type. Below we give $\operatorname{dim}(\operatorname{Der}(R))$ for every non-reduced Witt ring $R$ where the associated group has order at most 8 (cf. [5, p. 122-124]).

$\begin{array}{ll:lr}\mathbf{Z}_{2} & 0 & \mathbf{Z}_{4} \times{ }_{w} \mathbf{Z}_{4} \times{ }_{w} \mathbf{Z}_{4} & 6 \\ \mathbf{Z}_{4} & 0 & \mathbf{Z}_{4} \times{ }_{w} \mathbf{Z}_{4}\left[\Delta_{1}\right] & 5 \\ \mathbf{Z}_{2}\left[\Delta_{1}\right] & 2 & \mathbf{Z}_{4} \times{ }_{w} \mathbf{Z}_{2}\left[\Delta_{2}\right] & 8 \\ \mathbf{Z} \times{ } \mathbf{Z}_{4} & 1 & \mathbf{Z}_{2}\left[\Delta_{1}\right] \times{ }_{w} \mathbf{Z}_{2}\left[\Delta_{1}\right] \times{ }_{w} \mathbf{Z}_{2}\left[\Delta_{1}\right] & 9 \\ \mathbf{Z}_{4} \times{ }_{w} \mathbf{Z}_{4} & 2 & \mathbf{Z}_{2}\left[\Delta_{1}\right] \times{ }_{w} \mathbf{Z}_{2}\left[\Delta_{2}\right] & 10 \\ \mathbf{Z}_{2}\left[\Delta_{1}\right] \times{ }_{w} \mathbf{Z}_{2}\left[\Delta_{1}\right] & 4 & \left(\mathbf{Z} \times{ }_{w} \mathbf{Z}_{4}\right)\left[\Delta_{1}\right] & 4 \\ \mathbf{Z}_{4}\left[\Delta_{1}\right] & 2 & \left(\mathbf{Z}_{4} \times{ } \mathbf{Z}_{4}\right)\left[\Delta_{1}\right] & 8 \\ \mathbf{Z}_{2}\left[\Delta_{2}\right] & 8 & \left(\mathbf{Z}_{2}\left[\Delta_{1}\right] \times{ }_{w} \mathbf{Z}_{2}\left[\Delta_{1}\right]\right)\left[\Delta_{1}\right] & 14 \\ \mathbf{Z}\left[\Delta_{1}\right] \times{ }_{w} \mathbf{Z}_{4} & 2 & \mathbf{Z}_{4}\left[\Delta_{2}\right] & 8 \\ \mathbf{Z} \times{ }_{w} \mathbf{Z}_{4} \times{ }_{w} \mathbf{Z}_{4} & 4 & \mathbf{Z}_{2}\left[\Delta_{3}\right] & 28 \\ \mathbf{Z} \times{ }_{w} \mathbf{Z}_{4}\left[\Delta_{1}\right] & 3 & L_{3} & 5 \\ \mathbf{Z} \times{ }_{w} \mathbf{Z}_{2}\left[\Delta_{2}\right] & 6 & & \end{array}$

Any two reduced Witt rings have Lie isomorphic derivation algebras, namely 0 (1.4). To get a non-trivial example we consider the four Witt rings listed above with 2-dimensional derivation algebras.

Proposition 3.1. (1) $\left(\operatorname{Der}\left(\mathbf{Z}_{4}\left[\Delta_{1}\right]\right)\right.$ is abelian .

(2) $\operatorname{Der}\left(\mathbf{Z}_{2}\left[\Delta_{1}\right]\right), \operatorname{Der}\left(\mathbf{Z}_{4} \times{ }_{w} \mathbf{Z}_{4}\right)$ and $\operatorname{Der}\left(\mathbf{Z} \times{ }_{w} \mathbf{Z} \times{ }_{w} \mathbf{Z}_{4}\right)$ are nonabelian and Lie isomorphic.

Proof. Up to isomorphism there is a unique non-abelian 2-dimensional Lie algebra [2, p. 11], so we need only check if the algebras are abelian or not.

$\mathbf{Z}_{4}\left[\Delta_{1}\right]$ can be realized by $W\left(\mathbf{Z}_{3}((t))\right)$, hence by (1.5), $\operatorname{Der}\left(\mathbf{Z}_{4}\left[\Delta_{1}\right]\right)$ is generated by $d(\langle 1,1\rangle)$ and $d(t\langle 1,1\rangle$ ) (where $d(w)$ sends \pm 1 to 0 and $\pm t$ to $w)$. Then

$$
[d(\langle 1,1\rangle), d(t\langle 1,1\rangle)](t)=d(\langle 1,1\rangle)(t\langle 1,1\rangle)=t\langle\langle 1,1\rangle\rangle=0 .
$$

So $\operatorname{Der}\left(\mathbf{Z}_{4}\left[\Delta_{1}\right]\right)$ is abelian.

$\mathbf{Z}_{2}\left[\Delta_{1}\right]$ can be realized by $W\left(\mathbf{Z}_{5}\right)$. Let $\bar{a}=a+5 \mathbf{Z}$ for $a \in \mathbf{Z}$. Then $\operatorname{Der}\left(\mathbf{Z}_{2}\left[\Delta_{1}\right]\right)$ is generated by $d(\overline{1})$ and $d(\overline{2})$ (where $d(\bar{a})$ sends $\overline{1}$ to 0 and $\overline{2}$ to $\bar{a})$. Then $[d(\overline{1}), d(\overline{2})]=d(\overline{1})$ and $\operatorname{Der}\left(\mathbf{Z}_{2}\left[\Delta_{1}\right]\right)$ is non-abelian.

$\mathbf{Z}_{4} \times_{w} \mathbf{Z}_{4}$ can be realized by the Witt ring of the group $G=\{ \pm 1$, $\pm a\}$ with $D\langle 1, g\rangle=G$ for all $g \in G$. Using (1.8) we get $\operatorname{Der}\left(\mathbf{Z}_{4} \times{ }_{w} \mathbf{Z}_{4}\right)$ 
is generated by $d(\langle 1,1\rangle)$ and $d(\langle 1, a\rangle)$, where $d(w)$ sends \pm 1 to 0 and $\pm a$ to $w$. Then $[d(\langle 1,1\rangle), d(\langle 1, a\rangle)]=d(\langle 1,1\rangle)$ and the algebra is non-abelian.

Lastly, $\mathbf{Z} \times{ }_{w}, \mathbf{Z} \times{ }_{w} \mathbf{Z}_{4}$ can be realized by the Witt ring of the group $G=\{ \pm 1, \pm a, \pm b, \pm a b\} \quad$ with $D\langle 1,1\rangle=\{1,-a b\}, \quad D\langle 1, a\rangle=$ $\{1, a,-b,-a b\}$ and $D\langle 1, b\rangle=\{1, b,-a,-a b\}$. Using (1.8) we get $\operatorname{Der}\left(\mathbf{Z} \times{ }_{w} \mathbf{Z} \times{ }_{w} \mathbf{Z}_{4}\right)$ is generated by $D_{1}$ which sends $a$ to $\langle 1, a b\rangle, b$ to 0 , and by $D_{2}$ which sends $a$ to 0 and $b$ to $\langle 1, a b\rangle$. Then $\left[D_{1}, D_{2}\right]=D_{1}+D_{2}$ and the algebra is non-abelian.

Note that the Witt rings of (3.1)(2) with Lie isomorphic derivation algebras have non-isomorphic associated groups. However, it is possible for non-isomorphic Witt rings to have isomorphic associated groups and non-trivial Lie isomorphic derivation algebras. An example is $\mathbf{Z} \times{ }_{w} \mathbf{Z}_{2}\left[\Delta_{2}\right]$ and $\mathbf{Z}_{4} \times_{w} \mathbf{Z}_{4} \times_{w} \mathbf{Z}_{4}$. We sketch the computations required to verify this.

$\mathbf{Z}_{4} \times{ }_{w} \mathbf{Z}_{4} \times{ }_{w} \mathbf{Z}_{4}$ can be realized by the Witt ring of $G=$ $\{ \pm 1, \pm a, \pm b, \pm a b\}$, where $D\langle 1, g\rangle=G$ for all $g \in G$. The following is a basis for the derivation algebra:

$$
\begin{array}{ll}
D_{1}: a \mapsto\langle 1,1\rangle, b \mapsto 0, & D_{4}: a \mapsto 0, b \mapsto\langle 1,1\rangle, \\
D_{2}: a \mapsto\langle 1, a\rangle, b \mapsto 0, & D_{5}: a \mapsto 0, b \mapsto\langle 1, a\rangle, \\
D_{3}: a \mapsto\langle 1, b\rangle, b \mapsto 0, & D_{6}: a \mapsto\langle 1, a\rangle, b \mapsto\langle 1, b\rangle .
\end{array}
$$

$\mathbf{Z} \times{ }_{w} \mathbf{Z}_{2}\left[\Delta_{2}\right]$ can be realized by the Witt ring of the same $G$ but with $D\langle 1,1\rangle=\{1, a, b, a b\}, \quad D\langle 1, a\rangle=\{1, a\}, \quad D\langle 1, b\rangle=\{1, b\} \quad$ and $D\langle 1, a b\rangle=\{1, a b\}$. The following is a basis for the derivation algebra:

$$
\begin{array}{lll}
d_{1}: a \mapsto\langle\langle-a,-b\rangle\rangle, b \mapsto 0, & d_{4}: a \mapsto 0, b \mapsto\langle\langle-a, b\rangle\rangle, \\
d_{2}: a \mapsto\langle\langle-a, b\rangle\rangle, b \mapsto\langle 1,-b\rangle, & d_{5}: a \mapsto 0, b \mapsto b\langle 1,-a\rangle, \\
d_{3}: a \mapsto\langle 1,-b\rangle, b \mapsto 0, & d_{6}: a \mapsto b\langle 1,-a\rangle, b \mapsto\langle 1,-b\rangle .
\end{array}
$$

The map $D_{i} \mapsto d_{i}$ gives a Lie isomorphism from $\operatorname{Der}\left(\mathbf{Z}_{4} \times{ }_{w} \mathbf{Z}_{4} \times_{w} \mathbf{Z}_{4}\right)$ to $\left.\operatorname{Der} \mathbf{Z} \times{ }_{w} \mathbf{Z}_{2}\left[\Delta_{2}\right]\right)$ as can be easily, if not quickly, checked.

We close this section with an example of a derivation which arises naturally in the theory of quadratic forms. We use the following set-up: Let $F$ be a field of characteristic not $2, e \in \dot{F} \backslash \dot{F}^{2}$ and $E=F(\sqrt{e})$. Let - denote the involution on $E$ with $\overline{a+b \sqrt{e}}=a-b \sqrt{e}$, and also the induced involution on $W E$. Let $s: E \rightarrow F$ be the $F$-linear functional defined by $s(1)=0, s(\sqrt{e})=1$. We denote the Scharlau transfer of $s$ by $s_{*}$, and the map $W F \rightarrow W E$ induced by inclusion by $i_{*}$. 
LEMMA 3.2. Let $E=F(\sqrt{e})$ and $s^{*}=i_{*} s_{*}: W E \rightarrow W E$. Then for all $q_{1}, q_{2} \in W E:$

$$
s^{*}\left(q_{1} q_{2}\right)=\bar{q}_{1} s^{*}\left(q_{2}\right)+q_{2} s^{*}\left(q_{1}\right) .
$$

Proof. We need only check this for $x, y \in \dot{E}$ since $s^{*}$ is additive. Let $t: E \rightarrow F$ be the trace functional. If $z=\frac{1}{2} \sqrt{e}$ then $t(x z)=s(x)$ for all $x \in \dot{E}$. Let $t^{*}=i_{*} t_{*}$. By the trace formula of Scharlau-Knebusch [4, p. 212], $t^{*}(q)=q+\bar{q}$ for all $q \in W E$. We obtain:

$$
\begin{aligned}
\bar{y} s^{*}(x)+x s^{*}(y) & =\bar{y} t^{*}(x z)+x t^{*}(y z) \\
& =\bar{y}\langle x z, \overline{x z}\rangle+x\langle y z, \overline{y z}\rangle=\langle x y z, \overline{x y z}\rangle+x \bar{y}\langle z, \bar{z}\rangle \\
& =t^{*}(x y z)+x \bar{y}\left\langle\frac{1}{2} \sqrt{e},-\frac{1}{2} \sqrt{e}\right\rangle=s^{*}(x y) .
\end{aligned}
$$

COROllary 3.3. Let $E=F(\sqrt{e})$ and suppose that for all $x, y \in \dot{E}$ $N_{E / F}(y) \in D_{E}\left\langle 1,-N_{E / F}(x)\right\rangle$. Then $s^{*}=i_{*} s_{*}: W E \rightarrow W E$ is a derivation.

Proof. By an easy computation (cf. [4, p. 202]), if $x \in \dot{E}$ then $s^{*}(x)=z\left\langle 1,-N_{E / F}(x)\right\rangle$, for some $z \in \dot{F}$. By assumption, for all $y \in \dot{E}$, $\left\langle 1,-N_{E / F}(y)\right\rangle s^{*}(x)=0$ and so $\bar{y} s^{*}(x)=y s^{*}(x)$. The result thus follows from (3.2).

EXAMPLES. We give some examples of fields $F$ for which the condition of (3.3) is satisfied for all quadratic extensions.

(i) Finite fields. In this case binary forms represent all of $\dot{F}$.

(ii) Local fields. here either $\left\langle\left\langle-N_{E / F}(x),-N_{E / F}(y)\right\rangle\right\rangle$ is 0 in $W F$ or $\langle\langle-e,-f\rangle\rangle$ for some $f \in \dot{F} \backslash D_{F}\langle 1,-e\rangle$, since $W F$ has a unique non-trivial 2-fold Pfister form. In either case, $\left\langle\left\langle-N_{E / F}(x),-N_{E / F}(y)\right\rangle\right\rangle$ $\otimes E=0$ gives the condition of (3.3).

(iii) If the condition of (3.3) holds for every quadratic extension of $F_{1}$ and $F_{2}$ then it holds for every quadratic extension of the field $F$ constructed by Kula [3] with $W F=W F_{1} \times{ }_{w} W F_{2}$.

4. Simple derivation algebras. We begin with a simple observation:

LEMMA 4.1. Let $R$ be a Witt ring, $D \in \operatorname{Der}(R)$ and suppose $D\left(I_{R}\right) \subset$ $I_{R}^{m}$, for some $m \geq 1$. Then for all $k \geq 1, D\left(I_{R}^{k}\right) \subset I_{R}^{m+k-1}$.

Proof. We use induction on $k$, the case $k=1$ being trivial. Suppose $k>1$; we need only check the value of $D$ on $k$-fold Pfister forms.

$$
\begin{aligned}
D\left(\left\langle\left\langle a_{1}, \ldots, a_{k}\right\rangle\right\rangle\right)= & \left\langle 1, a_{k}\right\rangle D\left(\left\langle\left\langle a_{1}, \ldots, a_{k-1}\right\rangle\right\rangle\right) \\
& +\left\langle\left\langle a_{1}, \ldots, a_{k-1}\right\rangle\right\rangle D\left(\left\langle 1, a_{k}\right\rangle\right) .
\end{aligned}
$$


Now $D\left(\left\langle 1, a_{k}\right\rangle\right) \subset I_{R}^{m}$ and by induction $D\left(\left\langle\left\langle a_{1}, \ldots, a_{k-1}\right\rangle\right\rangle\right) \subset I_{R}^{m+k-2}$. Thus $D\left(\left\langle\left\langle a_{1}, \ldots, a_{k}\right\rangle\right\rangle\right) \in I_{R}^{m+k-1}$ as desired.

The generalized Witt algebra $W_{n}$ is the derivation algebra of $\mathbf{Z}_{2}\left[t_{1}, \ldots, t_{n}\right] /\left(t_{1}^{2}, \ldots, t_{n}^{2}\right)$ (cf. [7]). Since the Witt ring $\mathbf{Z}_{2}\left[\Delta_{n}\right]$ is isomorphic to $\mathbf{Z}_{2}\left[t_{1}, \ldots, t_{n}\right] /\left(t_{1}^{2}, \ldots, t_{n}^{2}\right)$ we have $W_{n}=\operatorname{Der}\left(\mathbf{Z}_{2}\left[\Delta_{n}\right]\right)$. We will show this is the only example of a simple derivation algebra of a finitely generated Witt ring.

THEOREM 4.2. Let $R$ be a finitely generated Witt ring and suppose $L=\operatorname{Der}(R) \neq 0$. The following are equivalent:

(1) $L$ is simple;

(2) L is semi-simple;

(3) L has no (non-zero) abelian ideals;

(4) $L \cong W_{n}$ and $R \cong \mathbf{Z}_{2}\left[\Delta_{n}\right]$, for some $n$.

Proof. We need only show (3) $\rightarrow$ (4). So suppose $L$ has no (non-zero) abelian ideals. Let $G$ be the group associated to $R$. Write $R=R_{0}[\Delta]$, for some group $\Delta$ of exponent 2 and Witt ring $R_{0}$ which is not a group ring. Let $G_{0}$ be the group associated to $R_{0}$.

Step 1. $I_{R_{0}}^{2}$ is torsion-free.

Suppose otherwise and choose $m \geq 2$ such that $I_{R_{0}}^{m}$ is not torsion-free but $I_{R_{0}}^{m+1}$ is torsion-free (this is possible as $R_{0}$ is finitely generated, cf. [5, 9.4]). Note that a torsion form in $I_{R_{0}}^{m}$ is universally round. Let $J=R \cdot I_{R_{0}}^{m}$ and $I=\{D \in L \mid D(R) \subset J\} . I \neq 0$ by (1.3). We will obtain a contradiction by showing $I$ is an abelian ideal.

Clearly $I$ is closed under addition. Choose $D \in I, D^{\prime} \in L$ and $g \in G$. By (1.5) $L=\Delta L_{1}+L_{2}$, where $L_{1}=\left\{D \in L \mid D(\Delta)=0, D\left(R_{0}\right)\right.$ $\left.\subset R_{0}\right\}$ and $L_{2}=\left\{D \in L \mid D\left(R_{0}\right)=0\right\}$. Since $R_{0}$ is not a group ring extension, (1.7) implies $D\left(R_{0}\right) \subset I_{R_{0}}$ for $D \in L_{1}$. Write $D^{\prime}=\left(\sum \delta_{i} D_{i}\right)+$ $D^{\prime \prime}$, with $\delta_{i} \in \Delta, D_{i} \in L_{1}$ and $D^{\prime \prime} \in L_{2}$. Write $D(g)=\sum \gamma_{i} \varphi_{i}$, with $\gamma_{i} \in \Delta$ and $\varphi_{i} \in I_{R_{0}}^{m}$. We obtain:

$$
\begin{aligned}
{\left[D, D^{\prime}\right](g) } & =D\left(D^{\prime}(g)\right)+\sum D^{\prime}\left(\gamma_{i} \varphi_{i}\right) \\
& =D\left(D^{\prime}(g)\right)+\sum \varphi_{i} D^{\prime}\left(\gamma_{i}\right)+\sum_{i} \gamma_{i}\left(\sum_{j} \delta_{j} D_{j}\left(\varphi_{i}\right)\right)+D^{\prime \prime}\left(\varphi_{i}\right) .
\end{aligned}
$$

Now $D\left(D^{\prime}(g)\right) \in J$ since $D \in I, \varphi_{i} D^{\prime}\left(\gamma_{i}\right) \in R \cdot I_{R_{0}}^{m}=J, D^{\prime \prime}\left(\varphi_{i}\right)=0$ since $D^{\prime \prime} \in L_{2}$ and $\gamma_{i} \delta_{j} D_{j}\left(\varphi_{i}\right) \in R \cdot I_{R_{0}}^{m}=J$ by (4.1). Hence $\left[D, D^{\prime}\right] \in I$ and $I$ is an ideal. 
Lastly, we show $I$ is abelian. If $D_{1}, D_{2} \in I$ and $g \in D$ then

$$
D_{1}\left(D_{2}(g)\right) \in D_{1}\left(R \cdot I_{R_{0}}^{m}\right) \subset R D_{1}\left(I_{R_{0}}^{m}\right)+I_{R_{0}}^{m} D_{1}(R) \subset R \cdot I_{R_{0}}^{2 m-1}
$$

by (4.1). Then since $m \geq 2,(1.1)$ implies $D_{1}\left(D_{2}(g)\right) \subset R \cdot I_{R_{0}}^{2 m-1} \cap R_{t}=$ 0 . Hence $[I, I]=0$.

\section{Step 2. $R \neq R_{0}$.}

Suppose $R=R_{0}$. If $I_{R}$ is torsion-free then either $R$ is reduced or $\mathbf{Z}_{2}$, and in both cases $L=\operatorname{Der}(R)=0$ by (1.4). So we may assume $I_{R}$ is not torsion-free, in particular, that $D\langle 1,1\rangle \neq\{1\}$. If $x \in D\langle 1,1\rangle$ then $\langle 1,-x\rangle \in \operatorname{ur}(R)$ since $\langle 1,-x\rangle$ is torsion and $I_{R}^{2}$ is torsion-free by Step 1 . We consider four cases. In each case we obtain a contradiction by construction of non-zero abelian ideal.

Case 1. $G \neq\{1,-1\} D\langle 1,1\rangle$ and $1 \neq-1$.

Fix a subgroup $H$ of index 2 in $G$ such that $H$ contains $\{1,-1\} D\langle 1,1\rangle$. Set $I=\{d(H,\langle 1,-x\rangle) \mid x \in D\langle 1,1\rangle\}$, using the notation of (1.3). $I \neq 0$ since $D\langle 1,1\rangle \neq\{1\}$ :

Now $d(H,\langle 1,-x\rangle)+d(H,\langle 1,-y\rangle)=d(H,\langle 1,-x y\rangle)$, so $I$ is closed under addition. Let $D \in L$ and $g \in G$. Then $D(g) \in \operatorname{ann}_{R}\langle 1,1\rangle$ $=I_{R} \cap R_{t}$, since $-1 \neq 1$. We can find an $e \in G$ such that $D(g)+$ $\langle 1,-e\rangle \in I_{R}^{2}$. Then $\langle\langle 1,-e\rangle\rangle=\langle 1,1\rangle D(g)+\langle\langle 1,-e\rangle\rangle \in I_{R}^{3}$ and so $\langle\langle 1,-e\rangle\rangle=0$. Thus $D(g)+\langle 1,-e\rangle \in I_{R}^{2} \cap R_{t}=0$, by Step 1 . Hence for every $g \in G$ there exists an $e \in D\langle 1,1\rangle$ such that $D(g)=\langle 1,-e\rangle$.

We now complete the proof that $I$ is an ideal. $[D, d(H,\langle 1,-x\rangle)](g)$ $=D(d(H,\langle 1,-x\rangle)(g))$, since $D(g)=\langle 1,-e\rangle$ with $e \in D\langle 1,1\rangle \subset H$. In particular, $[D, d(H,\langle 1,-x\rangle)](g)=0$ if $g \in H$. If $g \notin H$ and $D(x)=$ $\langle 1,-y\rangle$ for some $y \in D\langle 1,1\rangle$ then $[D, d(H,\langle 1,-x\rangle)](g)=D(\langle 1,-x\rangle)$ $=\langle 1,-y\rangle$. Hence $[D, d(H,\langle 1,-x\rangle)]=d(H,\langle 1,-y\rangle) \in I$.

Lastly, we show $I$ is abelian. Since the image of $d(H,\langle 1,-x\rangle)$ is $\{0$, $\langle 1,-x\rangle\}$ and $x \in D\langle 1,1\rangle \subset H$, the composition of any two derivations in $I$ is 0 . Thus $[I, I]=0$.

Case 2. $G=\{1,-1\} D\langle 1,1\rangle$ and $-1 \notin D\langle 1,1\rangle$.

For $g \in G$ define $D(g)=D\langle 1,-g\rangle$ if $g \in D\langle 1,1\rangle$, and $D(g)=$ $\langle 1, g\rangle$ if $-g \in D\langle 1,1\rangle$. Note that $D(g)=D(-g)$ for all $g$ and $D(-1)$ $=0$. To show $D \in L$ we need only check $D\left(g g^{\prime}\right)=D(g)+D\left(g^{\prime}\right)$ for all $g, g^{\prime} \in G$, since $D(g) \in \operatorname{ur}(R)$ for all $g \in G$. Now:

$$
\begin{aligned}
D(g) & \perp D\left(g^{\prime}\right)=\left\langle 1, \varepsilon_{1} g\right\rangle+\left\langle 1, \varepsilon_{2} g^{\prime}\right\rangle, \quad \text { for some } \varepsilon_{1}, \varepsilon_{2} \in\{1,-1\} \\
& =\left\langle-\varepsilon_{2} g^{\prime},-\varepsilon_{1} \varepsilon_{2} g g^{\prime}\right\rangle+\left\langle 1, \varepsilon_{2} g^{\prime}\right\rangle, \quad \text { as }\left\langle 1, \varepsilon_{1} g\right\rangle \in \operatorname{ur}(R) \\
& =\left\langle 1,-\varepsilon_{1} \varepsilon_{2} g g^{\prime}\right\rangle=D\left(g g^{\prime}\right) .
\end{aligned}
$$


Let $D^{\prime} \in L$ and $g \in G$ be arbitrary. As in Case $1 D^{\prime}(g)=\langle 1,-x\rangle$ for some $x \in D\langle 1,1\rangle$. Then

$$
\begin{aligned}
{\left[D^{\prime}, D\right](g) } & =D^{\prime}(\langle 1, \varepsilon g\rangle)+D(\langle 1,-x\rangle)=D^{\prime}(g)+D(x) \\
& =\langle 1,-x\rangle+\langle 1,-x\rangle=0 \quad(\text { where } \varepsilon \in\{1,-1\}) .
\end{aligned}
$$

Hence $\{0, D\}$ is an abelian ideal.

Case 3. $G=D\langle 1,1\rangle$ and $-1 \neq 1$.

Here we have $\langle 1,1\rangle \in \operatorname{ur}(R)$. Set $I=\{D \in L \mid D(R) \subset\{0,\langle 1,1\rangle\}\}$. $I \neq 0$ by (1.3). Since for any derivation $D^{\prime} \in L, D^{\prime}(1)=0$, we have for $D \in I$ that $\left[D, D^{\prime}\right](R) \subset D\left(D^{\prime}(R)\right) \subset\{0,\langle 1,1\rangle\}$. Thus $I$ is an ideal. Further, $I$ is abelian since the composition of any two derivations in $I$ is 0 .

Case 4. $-1=1$.

Since $R=R_{0}$ by assumption and $I_{R_{0}}^{2}$ is torsion-free by Step 1, we have $I_{R}^{2}=0$ and $\operatorname{ann}_{R}\langle 1,1\rangle=\{\langle 1, x\rangle \mid x \in G\} \subset \operatorname{ur}(R)$. We define $D$ : $G \rightarrow R$ by $D(x)=\langle 1, x\rangle$. Then $D(1)=0$ and $D(x y)=\langle 1, x y\rangle=\langle 1, x\rangle$ $+\langle 1, y\rangle=D(x)+D(y)=y D(x)+x D(y)$, since $\langle 1, x\rangle,\langle 1, y\rangle \in$ $\operatorname{ur}(R)$. So $D$ is a derivation. Further, for any $D^{\prime} \in L, g \in G$ we have $\left[D, D^{\prime}\right](g)=D\left(D^{\prime}(g)\right)+D^{\prime}(D(g))=D^{\prime}(g)+D^{\prime}(g)=0$ by (1.1). Thus $\{0, D\}$ is an abelian ideal.

Step 3. $I_{R_{0}}$ is torsion-free.

By Step 2, $|\Delta|=2^{n}$ with $n \geq 1$. Let $t_{1}, \ldots, t_{n}$ generate $\Delta$. By (1.5), $L=\Delta L_{1}+L_{2}$ where $L_{1}=\left\{D \in L \mid D(\Delta)=0, D\left(R_{0}\right) \subset R_{0}\right\}$ and $L_{2}=$ $\left\{D \in L \mid D\left(R_{0}\right)=0\right\}$. Note that if $D \in L_{1}$ then $D\left(R_{0}\right) \subset I_{R_{0}}$ by (1.7). For $\alpha_{1}, \ldots, \alpha_{n} \in \operatorname{ann}_{R}\langle 1,1\rangle$ let $d\left(\alpha_{1}, \ldots, \alpha_{n}\right)$ be the derivation sending $R_{0}$ to 0 and $t_{i}$ to $\alpha_{i}(1 \leq i \leq n)$. Then $L_{2}=\left\{d\left(\alpha_{1}, \ldots, \alpha_{n}\right) \mid \alpha_{i} \in\right.$ $\left.\operatorname{ann}_{R}\langle 1,1\rangle\right\}$.

Suppose $I_{R_{0}}$ is not torsion-free. Then $I_{R_{0}} \cap R_{t} \subset \operatorname{ann}_{R}\langle 1,1\rangle$, since $I_{R_{0}}^{2}$ is torsion-free by Step 1 . Set $J=R\left(I_{R_{0}} \cap R_{t}\right)$ and $I=$ $\left\{d\left(\alpha_{1}, \ldots, \alpha_{n}\right) \mid \alpha_{i} \in J\right\} . I$ is closed under addition. We will obtain a contradiction by showing $I$ is an abelian ideal.

First, if $\delta \in \Delta, D \in L_{1}$ and $d\left(\alpha_{1}, \ldots, \alpha_{n}\right) \in I$ then for $g \in G_{0}$ we have

$$
\begin{aligned}
& {\left[\delta D, d\left(\alpha_{1}, \ldots, \alpha_{n}\right)\right](g)=d\left(\alpha_{1}, \ldots, \alpha_{n}\right)(\delta D(g))} \\
& \quad=D(g) d\left(\alpha_{1}, \ldots, \alpha_{n}\right)(\delta) \in I_{R_{0}} \cdot R\left(I_{R_{0}} \cap R_{t}\right) \subset R\left(I_{R_{0}}^{2} \cap R_{t}\right)=0 .
\end{aligned}
$$


And $\left[\delta D, d\left(\alpha_{1}, \ldots, \alpha_{n}\right)\right]\left(t_{i}\right)=\delta D\left(\alpha_{i}\right) \in R\left(I_{R_{0}} \cap R_{t}\right)=J$. Hence $\left[\delta D, d\left(\alpha_{1}, \ldots, \alpha_{n}\right)\right]=d\left(\delta D\left(\alpha_{1}\right), \ldots, \delta D\left(\alpha_{n}\right)\right) \in I$ and $\left[\Delta L_{1}, I\right] \subset I$.

Next, choose $d\left(\beta_{1}, \ldots, \beta_{n}\right) \in L_{2}$ and $d\left(\alpha_{1}, \ldots, \alpha_{n}\right) \in I$. Write $\alpha_{i}=$ $\sum_{j} \delta_{i j} \varphi_{i j}$, for some $\delta_{i j} \in \Delta$ and $\varphi_{i j} \in I_{R_{0}} \cap R_{t}$. Then $\left[d\left(\beta_{1}, \ldots, \beta_{n}\right)\right.$, $\left.d\left(\alpha_{1}, \ldots, \alpha_{n}\right)\right]$ sends $R_{0}$ to 0 and sends $t_{i}$ to

$$
\begin{aligned}
d\left(\alpha_{1}, \ldots, \alpha_{n}\right)\left(\beta_{i}\right)+ & d\left(\beta_{1}, \ldots, \beta_{n}\right)\left(\alpha_{i}\right) \\
& =d\left(\alpha_{1}, \ldots, \alpha_{n}\right)\left(\beta_{i}\right)+\sum_{j} \varphi_{i j} d\left(\beta_{1}, \ldots, \beta_{n}\right)\left(\delta_{i j}\right) .
\end{aligned}
$$

Now $d\left(\alpha_{1}, \ldots, \alpha_{n}\right)\left(\beta_{i}\right) \in J$ and $\varphi_{i j} d\left(\beta_{1}, \ldots, \beta_{n}\right)\left(\delta_{i j}\right) \in\left(I_{R_{0}} \cap R_{t}\right) \cdot R=$ $J$. Thus $\left[L_{2}, I\right] \subset I$ and so $[L, I] \subset I$.

Lastly, to show $I$ is abelian choose $d\left(\alpha_{1}, \ldots, \alpha_{n}\right), d\left(\beta_{1}, \ldots, \beta_{n}\right) \in I$. Then $d\left(\alpha_{1}, \ldots, \alpha_{n}\right) \circ d\left(\beta_{1}, \ldots, \beta_{n}\right)$ sends $R_{0}$ to 0 and $t_{l}$ to $d\left(\alpha_{1}, \ldots, \alpha_{n}\right)\left(\beta_{i}\right)$. Write $\beta_{i}=\sum \delta_{j} \varphi_{j}$ with $\delta_{j} \in \Delta$ and $\varphi_{j} \in I_{R_{0}} \cap R_{t}$. Then

$$
\begin{aligned}
d\left(\alpha_{1}, \ldots, \alpha_{n}\right)\left(\beta_{i}\right) & =\sum_{j} \varphi_{j} d\left(\alpha_{1}, \ldots, \alpha_{n}\right)\left(\delta_{j}\right) \\
& \in\left(I_{R_{0}} \cap R_{t}\right) \cdot R\left(I_{R_{0}} \cap R_{t}\right) \subset R\left(I_{R_{0}}^{2} \cap R_{t}\right)=0 .
\end{aligned}
$$

Hence $\left[d\left(\alpha_{1}, \ldots, \alpha_{n}\right), d\left(\beta_{1}, \ldots, \beta_{n}\right)\right]=0$ and $[I, I]=0$.

We now complete the proof. We have $R=R_{0}\left[\Delta_{n}\right]$ with $n \geq 1$ and $I_{R_{0}}$ torsion-free. In particular, $D\langle 1,1\rangle=\{1\}$. Thus either $R_{0}$ is reduced or $R_{0} \cong \mathbf{Z}_{2}$. But if $R_{0}$ is reduced so is $R$ and $L=\operatorname{Der}(R)=0$. So $R_{0} \cong \mathbf{Z}_{2}, R \cong \mathbf{Z}_{2}\left[\Delta_{n}\right]$ and $L=\operatorname{Der}(R) \cong W_{n}$.

REMARK. Manin has shown [7, p. 106] that every restricted Lie algebra can be embedded in a generalized Witt algebra. Hence if $R$ is a finitely generated Witt ring there exists an $n$ such that $\operatorname{Der}(R)$ embeds in $\operatorname{Der}\left(\mathbf{Z}_{2}\left[\Delta_{n}\right]\right)$.

5. Integrable derivations. We follow the terminology of Matsumura [6]. If $R$ is a commutative ring with identity then a derivation $D$ of $R$ into itself is integrable if there exists a homomorphism $E: R \rightarrow R[[t]]$ such that $E(r) \equiv r+t D(r)\left(\bmod t^{2}\right)$ for all $r \in R$. The map $E$ is an integral of $D$. An integral $E$ arises from a collection of maps $\underline{D}=\left(D_{0}, D_{1}, D_{2}, \ldots\right)$ where $D_{0}=\mathrm{id}_{R}, D_{1}=D$ and $E(r)=\sum_{i} D_{\imath}(r) t^{i}$ for all $r \in R$. If $\underline{D}=$ $\left(1, D_{1}, D_{2}, \ldots\right)$ and $\underline{D}^{\prime}=\left(1, D_{1}^{\prime}, D_{2}^{\prime}, \ldots\right)$ yield integrals for the derivations $D$ and $D^{\prime}$ then:

$$
\underline{D D^{\prime}}=\left(1, D_{1}+D_{1}^{\prime}, D_{2}+D_{1} D_{1}^{\prime}+D_{2}^{\prime}, \ldots, \sum_{i} D_{\imath} D_{n-i}^{\prime}, \ldots\right)
$$


yields an integral for $D+D^{\prime}$. Indeed the set of integrable derivations form an $R$-submodule of $\operatorname{Der}(R)$.

Let $R$ be a Witt ring and let $D \in \operatorname{Der}(R)$. We say $D$ is $I_{R}$-integrable if $D$ has an integral $E$ such that $E(r)-r \in I_{R}[[t]]$ for all $r \in R$. Equivalently, we require $\underline{D}=\left(1, D_{1}, D_{2}, \ldots\right)$ to give an integral of $D$ with $D_{i}(R) \subset I_{R}$ for $i \geq 2$. The composition $D D^{\prime}$ above shows the set of $I_{R}$-integrable derivations also forms a subgroup of $\operatorname{Der}(R)$.

Lemma 5.1. Let $R$ be a Witt ring and $D \in \operatorname{Der}(R)$. If $D(R)^{2}=0$ then $D$ is $I_{R^{-}}$integrable.

Proof. $D(R)^{2}=0$ implies $D(R) \subset I_{R}$. Define $E: R \rightarrow R[[t]]$ by $E(r)=r+\sum_{i=1}^{\infty} D(r) t^{i} . E$ is additive, $E(r)-r \in I_{R}[[t]]$ and for $r, s \in$ $R_{i}$ :

$$
\begin{aligned}
E(r) E(s) & =r s+\sum_{i}\left(r D(s)+\sum_{j=1}^{i-1} D(r) D(s)+s D(r)\right) t^{i} \\
& =r s+\sum_{i} D(r s) t^{i}=E(r s),
\end{aligned}
$$

using that $D(r) D(s)=0$.

LEMMA 5.2. Let $R$ be $\mathbf{Z}_{2}, \mathbf{Z}_{4}$ or an indecomposable finitely generated Witt ring of local type. Then every derivation of $R$ is $I_{R^{-}}$integrable.

Proof. If $R$ is $\mathbf{Z}_{2}, \mathbf{Z}_{4}$ or $\mathbf{Z}$ then $\operatorname{Der}(R)=0$ (1.4). Suppose $R=L_{2 n, 1}$ or $L_{2 n-1}$ for $n \geq 2$. Then, by (2.4), $\operatorname{Der}(R)$ is generated by derivations of the form $d(H, \beta)$ (cf. (1.3)). Here $d(H, \beta)(R)=R \beta$, with $\beta \in \operatorname{ann}_{R}\langle 1,1\rangle$. Hence (2.2) implies $\beta$ is $y\langle 1, x\rangle$ or $\rho$, where $-x \in D\langle 1,1\rangle, y \in G$ and $\rho$ is the unique non-trivial 2-fold Pfister form of $R$. In either case $\beta^{2}=0$. So $d(H, \beta)$ is $I_{R}$-integrable by (5.1) and then every derivation of $R$ is $I_{R}$-integrable.

Now suppose $R=L_{2 n, 0}$ with $n \geq 2$. Let $G$ be the group associated to $R$ and fix $a_{0}, b_{0} \in G$ with $a_{0} \notin D\left\langle 1, b_{0}\right\rangle$. Write $G=A \times B$ with $a_{0} \in A$, $b_{0} \in B$ and satisfying $a_{1} \in D\left\langle 1, a_{2}\right\rangle, b_{1} \in D\left\langle 1, b_{2}\right\rangle$ for all $a_{1}, a_{2} \in A$, $b_{1}, b_{2} \in B$. This is possible by (2.12). Define $D_{A}$ on $G$ by $D_{A}(a b)=b\langle 1, \hat{a}\rangle$, with $a \in A, b \in B$. This is a derivation, as shown in the proof of (2.13). Note that $D_{A}(R)^{2}=\left\{\left\langle\left\langle a_{1}, a_{2}\right\rangle\right\rangle \mid a_{i} \in A\right\}=0$ so that $D_{A}$ is $I_{R^{-}}$-integrable by (5.1).

Let $D \in \operatorname{Der}(R)$. The proof of (2.13) shows that there exists a derivation $D_{1}$ in the subgroup generated by $D_{A}$ and $\{d(D\langle 1, x\rangle$, $\left.y\langle 1, x\rangle) \mid y \in G, x \in D\left\langle 1, b_{0}\right\rangle\right\}$ such that $D_{1}\left(a_{0}\right)=D\left(a_{0}\right)$ and $D_{1}\left(b_{0}\right)=0$. 
Further, $D_{1}$ is $I_{R}$-integrable. Similarly, there exists $I_{R}$-integrable $D_{2}$ such that $D_{2}\left(a_{0}\right)=0$ and $D_{2}\left(b_{0}\right)=D\left(b_{0}\right)$.

By replacing $D$ by $D-D_{1}-D_{2}$ if necessary, we see it suffices to show if $D \in L=\left\{D \in \operatorname{Der}(R) \mid D\left(a_{0}\right)=D\left(b_{0}\right)=0\right\}$ then $D$ is $I_{R}$-integrable. Now $L$ is isomorphic to $\operatorname{Der}\left(L_{2 n-1}\right)$ by (2.11). Let $\gamma_{*}: \operatorname{Der}\left(L_{2 n-1}\right)$ $\rightarrow L$ be an isomorphism (take the inverse of the map in (2.11)). The proof of $(2.11)$ showed that $\gamma_{*}(d(H, \alpha))=d(K, \beta)$, where $d(H, \alpha), d(K, \beta)$ are of the type discussed in (1.3). Thus the derivations $d(K, \beta) \in L$ generate $L$ by (2.4). As argued above, $d(K, \beta)$ is $I_{R}$-integrable. Hence $D$ is $I_{R}$-integrable.

LEMMA 5.3. Let $R_{0}$ be a (finitely generated) Witt ring and let $R=R_{0}\left[\Delta_{1}\right]$. Suppose every derivation of $R_{0}$ is $I_{R_{0}}$-integrable and $D \in$ $\operatorname{Der}(R)$ satisfies $D\left(R_{0}\right) \subset I_{R_{0}}$. Then $D$ is $I_{R^{-i n t e g r a b l e . ~}}$

Proof. Let $\Delta_{1}=\{1, t\}$. By (1.5) $D=D^{\prime}+t D^{\prime \prime}+D^{\prime \prime \prime}$, where $D^{\prime}, D^{\prime \prime}$ $\in L_{1}=\left\{D \in \operatorname{Der}(R) \mid D\left(\Delta_{1}\right)=0, \quad D\left(R_{0}\right) \subset R_{0}\right\} \quad$ and $\quad D^{\prime \prime \prime}\left(R_{0}\right)=0$. The restriction to $R_{0}$ gives an isomorphism between $L_{1}$ and $\operatorname{Der}\left(R_{0}\right)$ so

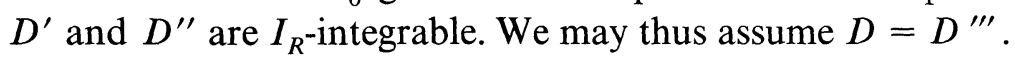

Let $w=D(t)$. By assumption, $w \in \operatorname{ann}_{R}\langle 1,1\rangle \cap I_{R}$. For $r \in R_{0}$, $D(r)=0$ and $D(r t)=r w$. If char $R=2$ then since $w^{2} \in 2 I_{R}=0, D(R)^{2}$ $=(w R)^{2}=0$ and $D$ is $I_{R}$-integrable by (5.1). So we may assume char $R$ $\neq 2$. For non-negative even integers $k$ we dfine $w_{k} \in I_{R}$ inductively by:

(i) $w_{0}=w$

(ii) If $k=4 i$ then $w_{2 i} \in I_{R}$ and $w_{2 i}^{2} \in 2 I_{R}$. Choose $w_{k} \in I_{R}$ such that:

$$
-2 t w_{k}=w_{2 i}^{2}+2\left(\sum_{j=1}^{i-1} w_{2 j} w_{k-2 \jmath}\right) .
$$

(iii) If $k=4 i+2$ then $w_{0}^{2} \in 2 I_{R}$. Choose a $w_{k} \in I_{R}$ such that:

$$
-2 t w_{k}=w_{0}^{2}+2\left(\sum_{j=1}^{i} w_{2 j} w_{k-2 j}\right) \text {. }
$$

We note the following identities:

$$
\begin{array}{ll}
-2 t w_{k}=\sum_{j=1}^{k / 2-1} w_{2 j} w_{k-2 j} & \text { if } k \equiv 0(\bmod 4), \\
-2 t w_{k}=w_{0}^{2}+\sum_{j=1}^{k / 2} w_{2 j} w_{k-2 j} & \text { if } k \equiv 2(\bmod 4) .
\end{array}
$$


For odd integers $k$, set $D_{k}=D$. Let $D_{0}=\mathrm{id}_{R}$ and for even $k \geq 2$ define $D_{k}: R \rightarrow R$ by $D_{k}\left(r_{1}+r_{2} t\right)=r_{2} w_{k}$, for all $r_{1}, r_{2} \in R_{0}$. Note that $D_{i}(R) \subset I_{R}$ for $i \geq 1$. Define $E: R \rightarrow R[[s]]$ by $E(r)=\sum D_{k}(r) s^{k}$. To show $E$ is the integral of $D$ it suffices to check for all $x, y \in G$ ( $G$ the group associated to $R$ ) that:

$$
D_{k}(x y)=\sum_{i=0}^{k} D_{i}(x) D_{k-i}(y) \text {. }
$$

Suppose $k$ is odd. Then $D_{k}(x y)=x D(y)+y D(x)=D_{0}(x) D_{k}(y)+$ $D_{0}(y) D_{k}(x)$. Thus $(*)$ becomes:

$$
0=D(x)\left(\sum_{j=1}^{(k-1) / 2} D_{2 j}(y)\right)+D(y)\left(\sum_{j=1}^{(k-1) / 2} D_{2 j}(x)\right) .
$$

This clearly holds if $x$ or $y$ are in $G_{0}$, the group associated to $R_{0}$. So suppose $x=g t, y=g^{\prime} t$ for some $g, g^{\prime} \in G_{0}$. Then:

$$
\begin{aligned}
D(x) & \left(\sum D_{2 j}(y)\right)+D(y)\left(\sum D_{2 j}(x)\right) \\
= & g w_{0}\left(g^{\prime} \sum w_{2 j}\right)+g^{\prime} w_{0}\left(g \sum w_{2 j}\right) \\
= & 2 g g^{\prime} w_{0}\left(\sum w_{2 j}\right)=0 .
\end{aligned}
$$

Now suppose $k$ is even. Let $\varepsilon_{k}=0$ if $k \equiv 0(\bmod 4)$ and $\varepsilon_{k}=1$ if $k \equiv 2(\bmod 4)$. Then $(*)$ becomes

$$
\text { (**) } \begin{aligned}
D_{k}(x y)= & \sum_{j \text { odd }} D_{j}(x) D_{k-j}(y)+\sum_{j \text { even }} D_{j}(x) D_{k-j}(y) \\
D_{k}(x y)= & \varepsilon_{k} D(x) D(y)+x D_{k}(y)+y D_{k}(x) \\
& +\sum_{j=1}^{k / 2-1} D_{2 j}(x) D_{k-2 j}(y) .
\end{aligned}
$$

The equation $(* *)$ holds if $x$ or $y$ are in $G_{0}$. So suppose $x=g t, y=g^{\prime} t$ for some $g, g^{\prime} \in G_{0}$. We want to show the right hand side of $(* *)$ is 0 since $D_{k}(x y)=D_{k}\left(g g^{\prime}\right)=0$. First consider the case $k \equiv 0(\bmod 4)$. The right hand side of $(* *)$ is:

$$
\begin{aligned}
g \operatorname{tg}^{\prime} w_{k}+g^{\prime} \operatorname{tg} w_{k}+\sum g w_{2 j} g^{\prime} w_{k-2 j} & =g g^{\prime}\left(2 t w_{k}+\sum w_{2 j} w_{k-2 j}\right) \\
& =0, \text { by construction of } w_{k} .
\end{aligned}
$$

If $k \equiv 2(\bmod 4)$ the right hand side of $(* *)$ is:

$$
\begin{array}{r}
g g^{\prime} w_{0}^{2}+g t g^{\prime} w_{k}+g^{\prime} t g w_{k}+g g^{\prime} \sum w_{2 j} w_{k-2 J} \\
=g g^{\prime}\left(w_{0}^{2}+2 t w_{k}+\sum w_{2 j} w_{k-2 J}\right) \\
=0, \text { again by construction of } w_{k} .
\end{array}
$$


LEMMA 5.4. Let $R_{1}, R_{2}$ be (finitely generated) Witt rings and let $R=R_{1} \times_{w} R_{2}$. Let $L_{i}^{*}=\left\{D \in \operatorname{Der}\left(R_{\imath}\right) \mid D\left(R_{i}\right) \subset I_{R_{l}}\right\}$ for $i=1,2$. Suppose every derivation in $L_{i}^{*}$ is $I_{R_{i}}$-integrable $(i=1,2)$. Then every derivation of $R$ is $I_{R}$-integrable.

Proof. Let $G_{i}$ be the group associated to $R_{i}(i=1,2) ; G=G_{1} \times G_{2}$ is the group associated to $R$. We use the notation of (1.8). If $D \in L_{1}$ then there exists $D^{\prime} \in L_{1}^{*}$ such that for all $\left(g_{1}, g_{2}\right) \in G, \quad D\left(g_{1}, g_{2}\right)=$ $\left(D^{\prime}\left(g_{1}\right), 0\right)$. Let $\underline{D}^{\prime}=\left(1, D_{1}^{\prime}, D_{2}^{\prime}, \ldots\right)$ yield an integral for $D^{\prime}$ with $D_{i}^{\prime}\left(R_{1}\right)$ $\subset I_{R_{1}}$ for $i \geq 2$. Define, for $i \geq 2, D_{i}: R \rightarrow R$ by $D_{l}\left(r_{1}, r_{2}\right)=\left(D_{i}^{\prime}\left(r_{1}\right), 0\right)$. Then $\underline{D}=\left(1, D, D_{2}, \ldots\right)$ yields an integral for $D$. Similarly, if $D \in L_{2}$ then $D$ is $I_{R}$-integrable.

If $D$ is in $E_{1}, E_{2}$ or $F$ then $D(R) \subset \operatorname{ur}(R)$ by (1.8). Hence, $D(R)^{2}=0$ and $D$ is $I_{R}$-integrable by (5.1). Since $L_{1}, L_{2}, E_{1}, E_{2}$ and $F$ generate $\operatorname{Der}(R)$, by (1.8), we have that every derivation on $R$ is $I_{R}$-integrable.

TheOREM 5.5. Let $R$ be a finitely generated Witt ring of elementary type. The following are equivalent:

(1) There exists $D \in \operatorname{Der}(R)$ with $D\left(I_{R}\right) \not \subset I_{R}$;

(2) There exists $D \in \operatorname{Der}(R)$ that is not integrable;

(3) There exists $D \in \operatorname{Der}(R)$ that is not $I_{R^{-}}$integrable;

(4) $R$ is a group ring and $\operatorname{char}(R)=2$.

Proof. (1) $\leftrightarrow$ (4) is (1.7) and (2) $\rightarrow$ (3) is clear.

(4) $\rightarrow(2)$ : Write $R=R_{0}\left[\Delta_{1}\right]$ with $\Delta_{1}=\{1, t\}$. There is a derivation $D$ with $D\left(R_{0}\right)=0$ and $D(t)=1$ (cf. (1.5)). Suppose $\underline{D}=\left(1, D, D_{2}, \ldots\right)$ yields an integral of $D$. Then $D_{2}(1)=0$ and

$$
\begin{aligned}
& 0=D_{2}(t \cdot t)=t D_{2}(t)+D(t) D(t)+t D_{2}(t) \\
& 0=\langle 1\rangle+2 t D_{2}(t),
\end{aligned}
$$

which is impossible, as $2 t D_{2}(t) \in I_{R}$ but $\langle-1\rangle \notin I_{R}$.

$(3) \rightarrow(4)$ : We use induction on $|G|$, where $G$ is the group associated to $R$. By (5.2), $R$ is not indecomposable. Suppose $R$ is a product $R_{1} \times{ }_{w} R_{2} \times{ }_{w} \cdots \times \times_{w} R_{n}$, with each $R_{i}$ indecomposable or a group ring. If $R_{i}$ is indecomposable then every derivation on $R_{l}$ is $I_{R_{l}}$-integrable. If $R_{i}$ is a group ring then $R_{i}=S_{i}[\Delta]$ with $S_{i}$ indecomposable or a Witt product. By induction, every derivation on $S_{l}$ is $I_{S_{l}}$-integrable. Hence any derivation on $R_{i}$ mapping $I_{R_{l}}$ into itself is $I_{R_{l}}$-integrable (5.3). Then (5.4) implies every derivation on $R$ is $I_{R}$-integrable, a contradiction. 
We thus have that $R$ is a group ring extension. Write $R=S[\Delta]$, with $S$ indecomposable or a Witt product. Again induction yields that every derivation on $S$ is $I_{S}$-integrable. If $\operatorname{char}(R) \neq 2$ then any derivation on $R$ maps $I_{R}$ into itself (1.7). So (5.3) implies every derivation on $R$ is $I_{R}$-integrable, contrary to our assumption. Thus $R$ is a group ring and $\operatorname{char}(R)=2$.

\section{REFERENCES}

[1] A. Carson and M. Marshall, Decomposition of Witt rings, Canad. J. Math., 34 (6) (1982), 1276-1302.

[2] N. Jacobson, Lie Algebras, Dover Pub., New York, 1979.

[3] M. Kula, Fields with prescribed quadratic form schemes, Math. Zeit., 167 (1979), 201-212.

[4] T. Y. Lam, The Algebraic Theory of Quadratic Forms, W. A. Benjamin, Reading, Massachusetts, 1973.

[5] M. Marshall, Abstract Witt Rings, Queen's Papers in Pure and Applied Math. 57, Queen's University, Kingston, Ontario, 1980.

[6] H. Matsumura, Integrable derivations, Nagoya Math. J., 87 (1982), 227-245.

[7] G. Seligman, Modular Lie Algebras, Springer, New York, 1967.

Received March 28, 1986.

SOUTHERN ILLINOIS UNIVERSITY

Carbondale, IL 62901 



\title{
PACIFIC JOURNAL OF MATHEMATICS EDITORS
}

\author{
V. S. VARADARAJAN \\ (Managing Editor) \\ University of California \\ Los Angeles, CA 90024 \\ HERBERT Clemens \\ University of Utah \\ Salt Lake City, UT 84112 \\ R. FINN \\ Stanford University \\ Stanford, CA 94305
}

\author{
HERMANN FLASCHKA \\ University of Arizona \\ Tucson, AZ 85721 \\ RAMESH A. GANGOLLI \\ University of Washington \\ Seattle, WA 98195 \\ VAUGHAN F. R. JONES \\ University of California \\ Berkeley, CA 94720 \\ ROBION KIRBY \\ University of California \\ Berkeley, CA 94720
}

\author{
C. C. Moore \\ University of California \\ Berkeley, CA 94720
}

H. SAMELSON

Stanford University

Stanford, CA 94305

HAROLD STARK

University of California, San Diego

La Jolla, CA 92093

\section{ASSOCIATE EDITORS}

\author{
R. ARENS \\ E. F. BECKENBACH \\ B. H. NEUMANN \\ F. WOLF \\ K. YOSHIDA \\ (1906-1982)

\section{SUPPORTING INSTITUTIONS} \\ UNIVERSITY OF ARIZONA \\ UNIVERSITY OF BRITISH COLUMBIA \\ CALIFORNIA INSTITUTE OF TECHNOLOGY \\ UNIVERSITY OF CALIFORNIA \\ MONTANA STATE UNIVERSITY \\ UNIVERSITY OF NEVADA, RENO \\ NEW MEXICO STATE UNIVERSITY \\ OREGON STATE UNIVERSITY

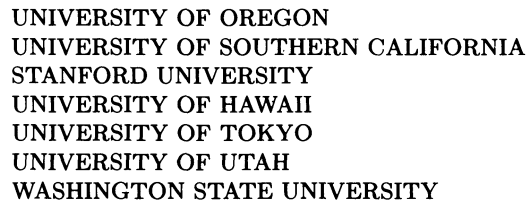

The Supporting Institutions listed above contribute to the cost of publication of this Journal, but they are not owners or publishers and have no responsibility for its content or policies.

Mathematical papers intended for publication in the Pacific Journal of Mathematics should be in typed form or offset-reproduced (not dittoed), double spaced with large margins. Please do not use built up fractions in the text of the manuscript. However, you may use them in the displayed equations. Underline Greek letters in red, German in green, and script in blue. The first paragraph must be capable of being used separately as a synopsis of the entire paper. In particular it should contain no bibliographic references. Please propose a heading for the odd numbered pages of less than 35 characters. Manuscripts, in triplicate, may be sent to any one of the editors. Please classify according to the scheme of Math. Reviews, Index to Vol. 39. Supply name and address of author to whom proofs should be sent. All other communications should be addressed to the managing editor, or Elaine Barth, University of California, Los Angeles, California 90024.

There are page-charges associated with articles appearing in the Pacific Journal of Mathematics. These charges are expected to be paid by the author's University, Government Agency or Company. If the author or authors do not have access to such Institutional support these charges are waived. Single authors will receive 50 free reprints; joint authors will receive a total of $\mathbf{1 0 0}$ free reprints. Additional copies may be obtained at cost in multiples of 50 .

The Pacific Journal of Mathematics is issued monthly as of January 1966. Regular subscription rate: $\$ 190.00$ a year (5 Vols., 10 issues). Special rate: $\$ 95.00$ a year to individual members of supporting institutions.

Subscriptions, orders for numbers issued in the last three calendar years, and changes of address should be sent to Pacific Journal of Mathematics, P.O. Box 969, Carmel Valley, CA 93924, U.S.A. Old back numbers obtainable from Kraus Periodicals Co., Route 100, Millwood, NY 10546.

The Pacific Journal of Mathematics at P.O. Box 969, Carmel Valley, CA 93924 (ISSN 0030-8730) publishes 5 volumes per year. Application to mail at Second-class postage rates is pending at Carmel Valley, California, and additional mailing offices. Postmaster: send address changes to Pacific Journal of Mathematics, P.O. Box 969, Carmel Valley, CA 93924.

PUBLISHED BY PACIFIC JOURNAL OF MATHEMATICS, A NON-PROFIT CORPORATION Copyright (C) 1987 by Pacific Journal of Mathematics 


\section{Pacific Journal of Mathematics}

Vol. 128 , No. $2 \quad$ April, 1987

Pierre Barrucand, John Harold Loxton and Hugh C. Williams, Some

explicit upper bounds on the class number and regulator of a cubic field

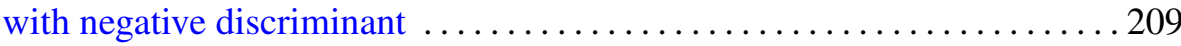

Thomas Ashland Chapman, Piecewise linear fibrations $\ldots \ldots \ldots \ldots \ldots 223$

Yves Félix and Jean-Claude Thomas, Extended Adams-Hilton's

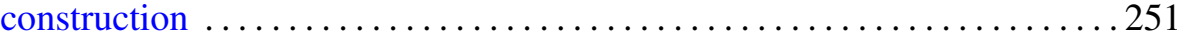

Robert Fitzgerald, Derivation algebras of finitely generated Witt rings . . . 265

K. Gopalsamy, Oscillatory properties of systems of first order linear delay differential inequalities ................................ 299

John P. Holmes, One parameter subsemigroups in locally complete

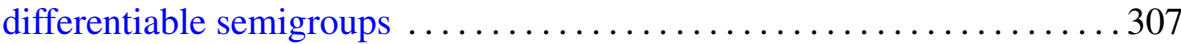

Douglas Murray Pickrell, Decomposition of regular representations for

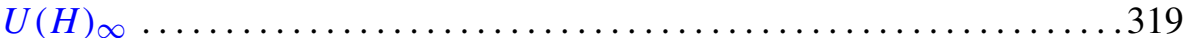

Victoria Powers, Characterizing reduced Witt rings of higher level

Parameswaran Sankaran and Peter Zvengrowski, Stable parallelizability

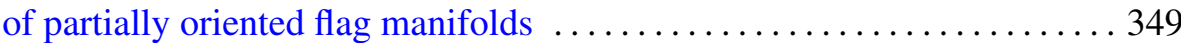

Johan Tysk, Eigenvalue estimates with applications to minimal surfaces . . . 361 Akihito Uchiyama, On McConnell's inequality for functionals of subharmonic functions

Minato Yasuo, Bott maps and the complex projective plane: a construction

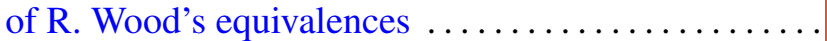

James Juei-Chin Yeh, Uniqueness of strong solutions to stochastic differential equations in the plane with deterministic boundary process 